ISSN 1420-3049

www.mdpi.com/journal/molecules

Article

\title{
Modular Synthesis of Polyphenolic Benzofurans, and Application in the Total Synthesis of Malibatol A and Shoreaphenol
}

\section{David Y.-K. Chen *, Qiang Kang and T. Robert Wu}

Chemical Synthesis Laboratory at Biopolis, Institute of Chemical and Engineering Sciences (ICES), Agency for Science, Technology and Research (A*STAR), 11 Biopolis Way, The Helios Block, No. 03-08, 138667, Singapore

* Author to whom correspondence should be addressed; E-Mail: david_chen@ices.a-star.edu.sg; Tel.: +65-6799-8502; Fax: +65-6874-5869.

Received: 29 July 2010; in revised form: 26 August 2010 / Accepted: 27 August 2010 / Published: 27 August 2010

\begin{abstract}
A modular strategy for the synthesis of hexacyclic dimeric resveratrol polyphenolic benzofurans is reported. The developed synthetic technology was applied to the total synthesis of malibatol A, shoreaphenol, and other biologically relevant polyphenols.
\end{abstract}

Keywords: cascade reaction; polyphenol; resveratrol; Friedel-Crafts; natural product; total synthesis

\section{Introduction}

Polyphenolic secondary metabolites have attracted growing interest from the scientific community in recent years [1-6]. However, despite their fascinating molecular architectures and diverse biological properties, chemical syntheses of these natural products and/or designed analogues have been scarce $[7,8]$. With this in mind, and as a continuation of our chemical and biological investigations of polyphenolic natural products $[9,10]$, we set out to develop a general strategy for the synthesis of dimeric, resveratrol-derived benzofurans represented by generic structure 1, as shown in Figure 1. We further demonstrated the developed technology in the total synthesis of malibatol A (2) and 
shoreaphenol (3), two dimeric resveratrol polyphenolic benzofurans isolated from Hopea malibato and Shorea robusta, respectively [11-13].

Figure 1. Generic molecular structure of polyphenolic benzofuran 1 and structures of malibatol A (2) and shoreaphenol (3).

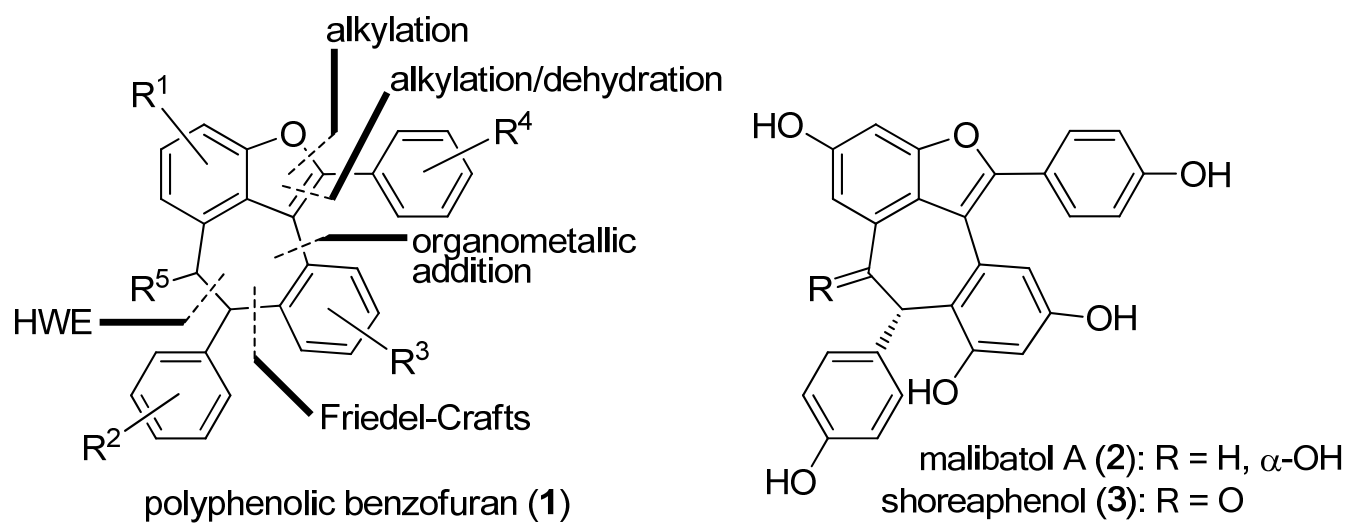

\section{Results and Discussion}

Recognizing the hexacyclic structure represented by $\mathbf{1}$ containing four substituted phenyl rings, we envisaged a modular approach where each one of the phenyl rings can be installed independently and sequentially. Therefore, as outlined in Scheme 1, the proposed synthesis begin with stilbene aldehyde 4, a building block with two aromatic domains brought together through a Horner-Wadswoth-Emmons (HWE) olefination reaction [14] and a subsequent Vilsmeier formylation [15]. Introduction of a third aromatic domain through the addition of an organometallic aryl species 5 to aldehyde $\mathbf{4}$, followed by subsequent oxidation (IBX) should give ketone 6. The intermediate benzylic alcohol obtained prior to IBX oxidation has previously been demonstrated by Snyder and co-workers as a versatile intermediate to access a number of resveratrol derived natural products [7,8]. Carbonyl-directed selective demethylation of 6 should lead to phenol 7, setting the stage for the attachment of the final aromatic moiety through an alkylation with benzyl halide 8 or a Mitsunobu reaction [16] with benzyl alcohol 9. With benzyl ether $\mathbf{1 0}$ in hand, the formation of the benzofuran ring is anticipated through its initial benzylic deprotonation (LiTMP), followed by an intramolecular cyclization (11 to 12) and subsequent dehydration (12 to $13, p-\mathrm{TsOH} \cdot \mathrm{H}_{2} \mathrm{O}$ ), to deliver pentacyclic benzofuran 13 [17]. Finally, the olefinic functionality in stilbene $\mathbf{1 3}$ should serve as a versatile handle for either direct seven-membered ring formation, or further transformation (14, e.g. epoxidation) leading to functionalized hexacycles 1 upon ring closure.

With this general strategy in mind, its realization to generate a library of benzofuran polyphenols is illustrated in Tables 1-3. As shown in Table 1, aryl ketones $\mathbf{1 6}$ and benzyl ethers $\mathbf{1 7}$ were efficiently prepared in $85-90 \%$ yield (over the two steps from 15) and $71-95 \%$ yield (over the two steps from 16), respectively. Next, benzofuran formation from keto benzyl ethers $\mathbf{1 7}$ under the two-step procedure generally proceeded in good yields (71-85\% yield, Table 2), apart from the failure of p-bromo substrate to participate in the cyclization (entry 3, Table 2) and the less satisfactory dehydration for the acid sensitive furanyl substrate (entry 5 , Table 2). 
Scheme 1. General, modular strategy for the construction of hexacyclic benzofuran 1

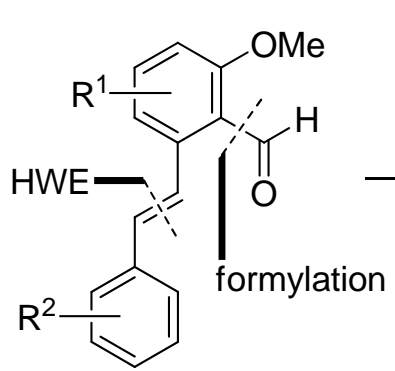

4 a)<smiles>[R]c1ccc([R5])cc1</smiles>

b) IBX

c) $\mathrm{BCl}_{3} \longrightarrow$ 6: $\mathrm{R}=\mathrm{Me}$<smiles>[R][R]1ccc(/C=C/c2ccccc2[R])c(C(=O)c2ccc([R])cc2)c1[R]</smiles>

d)

d)

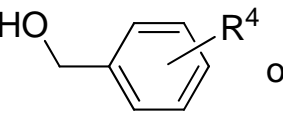

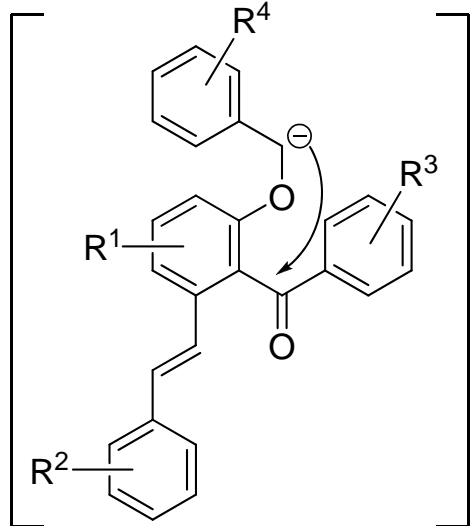

11 e)

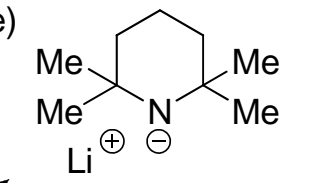

8: $(\mathrm{X}=\mathrm{Cl}, \mathrm{Br})$<smiles>[R9]CCCc1ccc([R])cc1</smiles><smiles>[R][R]1ccc(/C=C/c2cccc([2H])c2)c(C(=O)c2cccc([R])c2)c1OC(C)C=CC</smiles>

10<smiles>[R]CC(C)Cc1cccc2c1C(O)(c1ccc([R])cc1)C(c1ccc([R])cc1)O2</smiles>

12<smiles>[R]Cc1ccc2c3c(c(-c4ccc([R])cc4)oc13)-c1ccc([R])cc1C(c1cccc([R])c1)C2[R]</smiles>

h) ring closure

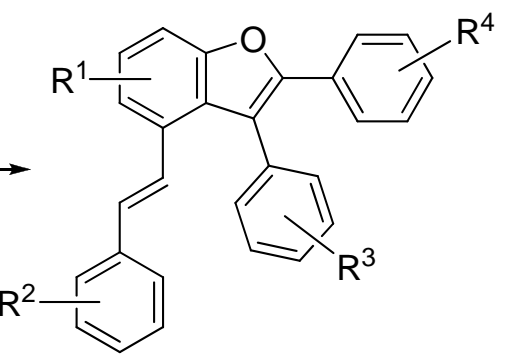

13

g) functionalization<smiles>[R][R]c1cccc(C=Cc2cccc3oc(-c4ccc([R])cc4)c(-c4ccccc4)c23)c1</smiles>

$\mathrm{Fn}=$ functional group 
Table 1. Preparation of ketone 16 and benzyl ether 17.

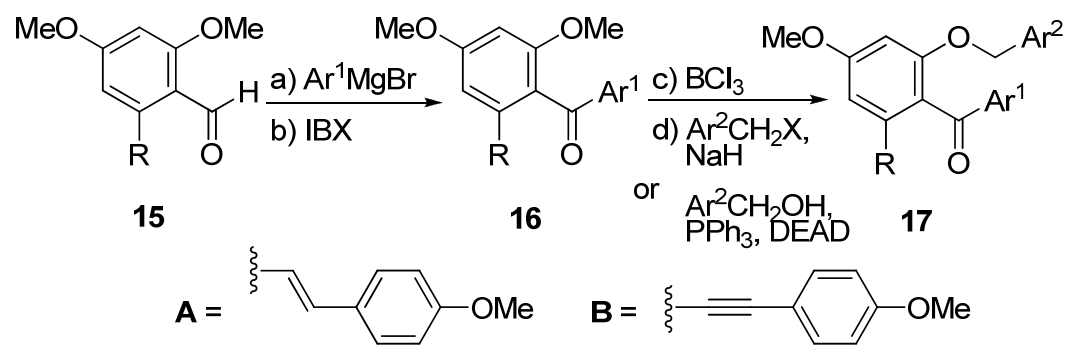

\begin{tabular}{|c|c|c|c|c|c|}
\hline Entry & $\mathbf{R}$ & $\mathbf{A r}^{1}$ & $\mathbf{A r}^{2}$ & 16 Yield (\%) ${ }^{b}$ & 17 Yield $(\%)^{b}$ \\
\hline $1(\mathrm{a})$ & $\mathrm{H}$ & $\mathrm{C}_{6} \mathrm{H}_{5}$ & $\mathrm{C}_{6} \mathrm{H}_{5}$ & $85 \%$ & $89 \%$ \\
\hline $2(b)$ & A & $3,5-(\mathrm{MeO})_{2} \mathrm{C}_{6} \mathrm{H}_{3}$ & $\mathrm{C}_{6} \mathrm{H}_{5}$ & $88 \%$ & $95 \%$ \\
\hline $3(\mathrm{c})$ & A & $3,5-(\mathrm{MeO})_{2} \mathrm{C}_{6} \mathrm{H}_{3}$ & 4- $(\mathrm{Br}) \mathrm{C}_{6} \mathrm{H}_{4}$ & $88 \%$ & $92 \%$ \\
\hline $4(d)$ & $\mathrm{A}$ & $3,5-(\mathrm{MeO})_{2} \mathrm{C}_{6} \mathrm{H}_{3}$ & 4-( $\mathrm{MeO}) \mathrm{C}_{6} \mathrm{H}_{4}$ & $88 \%$ & $90 \%$ \\
\hline $5(\mathrm{e})^{a}$ & A & $3,5-(\mathrm{MeO})_{2} \mathrm{C}_{6} \mathrm{H}_{3}$ & 2-furyl & $88 \%$ & $71 \%$ \\
\hline $6(f)$ & $\mathrm{A}$ & $\mathrm{C}_{6} \mathrm{H}_{5}$ & 4- $(\mathrm{MeO}) \mathrm{C}_{6} \mathrm{H}_{4}$ & $85 \%$ & $91 \%$ \\
\hline $7(\mathrm{~g})$ & A & $3,4,5-(\mathrm{MeO})_{3} \mathrm{C}_{6} \mathrm{H}_{2}$ & 4-( $(\mathrm{MeO}) \mathrm{C}_{6} \mathrm{H}_{4}$ & $90 \%$ & $95 \%$ \\
\hline $8(\mathrm{~h})$ & $\mathrm{A}$ & $3,4-(\mathrm{MeO})_{2} \mathrm{C}_{6} \mathrm{H}_{3}$ & 4-( $\mathrm{MeO}) \mathrm{C}_{6} \mathrm{H}_{4}$ & $87 \%$ & $90 \%$ \\
\hline 9(i) & $\mathrm{B}$ & $3,5-(\mathrm{MeO})_{2} \mathrm{C}_{6} \mathrm{H}_{3}$ & 4- $(\mathrm{MeO}) \mathrm{C}_{6} \mathrm{H}_{4}$ & $86 \%$ & $87 \%$ \\
\hline
\end{tabular}

Reagents and conditions: (a) $\mathrm{Ar}^{1} \mathrm{MgBr}$ (1.5 equiv), THF, $0{ }^{\circ} \mathrm{C}, 0.5 \mathrm{~h}$; (b) IBX (2.0 equiv), DMSO, $23{ }^{\circ} \mathrm{C}, 2 \mathrm{~h}$; (c) $\mathrm{BCl}_{3}\left(1.0 \mathrm{M}\right.$ in $\mathrm{CH}_{2} \mathrm{Cl}_{2}, 1.5$ equiv), $\mathrm{CH}_{2} \mathrm{Cl}_{2}, 0{ }^{\circ} \mathrm{C}, 1 \mathrm{~h}$; (d) $\mathrm{NaH}$ (2.0 equiv), $\mathrm{Ar}^{2} \mathrm{CH}_{2} \mathrm{X}$ (entry $\tilde{1} 3, \mathrm{X}=\mathrm{Br}$; entry $4, \tilde{6} 9, \mathrm{X}=\mathrm{Cl}$; 1.4 equiv), $\mathrm{DMF}, 0{ }^{\circ} \mathrm{C}$. ${ }^{a}$ furanyl alcohol (3.0 equiv), $\mathrm{PPh}_{3}$ (3.0 equiv), DEAD (3.0 equiv), THF, $0 \rightarrow 23{ }^{\circ} \mathrm{C}, 12 \mathrm{~h}$. ${ }^{b}$ Yields refer to chromatographically and spectroscopically homogeneous material. $\mathrm{DMF}=N, N^{\prime}$-dimethylformamide, $\mathrm{IBX}=0$ iodoxybenzoic acid; DEAD = diethyl azodicarboxylate.

Table 2. Preparation of benzofuran 19.
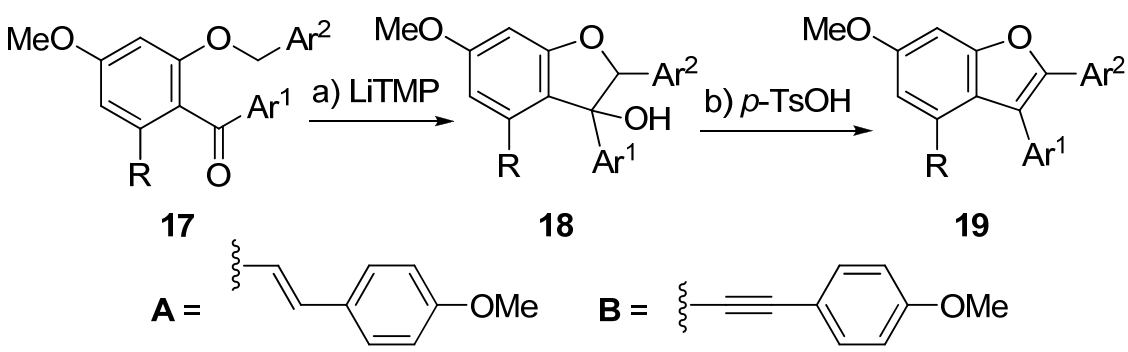

18 19

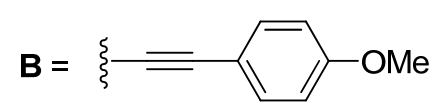

\begin{tabular}{lllll}
\hline Entry & $\mathbf{R}$ & $\mathbf{A r}$ & $\mathbf{A r}^{\mathbf{1}}$ & $\mathbf{1 9}^{\mathbf{1}}$ \\
\hline $1(\mathrm{a})$ & $\mathrm{H}$ & $\mathrm{C}_{6} \mathrm{H}_{5}$ & $\mathrm{C}_{6} \mathrm{H}_{5}$ & 83 \\
$2(\mathrm{~b})$ & A & $3,5-(\mathrm{MeO})_{2} \mathrm{C}_{6} \mathrm{H}_{3}$ & $\mathrm{C}_{6} \mathrm{H}_{5}$ & 71 \\
$3(\mathrm{c})$ & A & $3,5-(\mathrm{MeO})_{2} \mathrm{C}_{6} \mathrm{H}_{3}$ & $4-(\mathrm{Br}) \mathrm{C}_{6} \mathrm{H}_{4}$ & 0 \\
$4(\mathrm{~d})$ & A & $3,5-(\mathrm{MeO})_{2} \mathrm{C}_{6} \mathrm{H}_{3}$ & $4-(\mathrm{MeO}) \mathrm{C}_{6} \mathrm{H}_{4}$ & 87 \\
$5(\mathrm{e})$ & A & $3,5-(\mathrm{MeO})_{2} \mathrm{C}_{6} \mathrm{H}_{3}$ & $2-$ furyl & 38 \\
$6(\mathrm{f})$ & A & $\mathrm{C}_{6} \mathrm{H}_{5}$ & $4-(\mathrm{MeO}) \mathrm{C}_{6} \mathrm{H}_{4}$ & 80 \\
$7(\mathrm{~g})$ & A & $3,4,5-(\mathrm{MeO})_{3} \mathrm{C}_{6} \mathrm{H}_{2}$ & $4-(\mathrm{MeO}) \mathrm{C}_{6} \mathrm{H}_{4}$ & 85 \\
$8(\mathrm{~h})$ & A & $3,4-(\mathrm{MeO})_{2} \mathrm{C}_{6} \mathrm{H}_{3}$ & $4-(\mathrm{MeO}) \mathrm{C}_{6} \mathrm{H}_{4}$ & 81 \\
$9(\mathrm{i})$ & B & $3,5-(\mathrm{MeO})_{2} \mathrm{C}_{6} \mathrm{H}_{3}$ & $4-(\mathrm{MeO}) \mathrm{C}_{6} \mathrm{H}_{4}$ & 85 \\
\hline
\end{tabular}

Reagents and conditions: (a) LiTMP $\left(0.5 \mathrm{M}\right.$ in THF, 5 equiv), THF, $0{ }^{\circ} \mathrm{C}, 2 \mathrm{~h}$; (b) $p$ - $\mathrm{TsOH} \cdot \mathrm{H}_{2} \mathrm{O}(1.0$ equiv), $\mathrm{CH}_{2} \mathrm{Cl}_{2}, 23{ }^{\circ} \mathrm{C}, 1 \mathrm{~h} .{ }^{a}$ Yields refer to chromatographically and spectroscopically homogeneous material. LiTMP = Lithium 2,2,6,6-tetramethylpiperidide; $p$-TsOH = toluenesulfonic acid. 
Finally, closure of the seven-membered ring was carried out under acidic conditions $\left(p-\mathrm{TsOH} \cdot \mathrm{H}_{2} \mathrm{O}\right)$ to give cyclized compound 20 in high yields (90-95\% yield, entries 1, 2, 5-7, Table 3). The incompatibility of the furanyl functionality under the acidic conditions was once again observed (entry 3 , Table 3), and the electronically less favoured substrate 19d failed to participate in the Friedel-Crafts type cyclization (entry 4, Table 3 ).

Table 3. Friedel-Crafts type cyclization of benzofurans 20

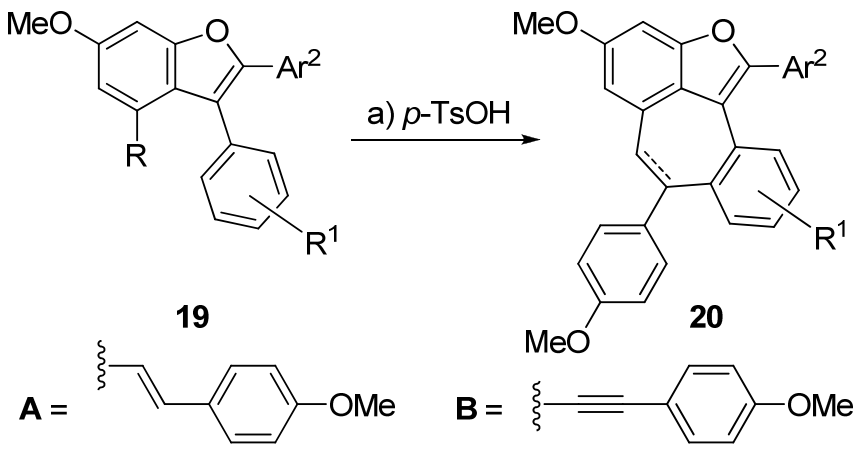

\begin{tabular}{ccllc}
\hline Entry & $\mathbf{R}$ & \multicolumn{1}{c}{$\mathbf{R}^{\mathbf{1}}$} & \multicolumn{1}{c}{$\mathbf{A r}^{\mathbf{2}}$} & 20 Yield (\%) \\
\hline $1(\mathrm{a})$ & A & $3,5-(\mathrm{MeO})_{2}$ & $\mathrm{C}_{6} \mathrm{H}_{5}$ & 95 \\
$2(\mathrm{~b})$ & A & $3,5-(\mathrm{MeO})_{2}$ & $4-(\mathrm{MeO}) \mathrm{C}_{6} \mathrm{H}_{4}$ & 90 \\
$3(\mathrm{c})$ & A & $3,5-(\mathrm{MeO})_{2}$ & $2-$ furyl & 0 \\
$4(\mathrm{~d})$ & A & $\mathrm{H}$ & $4-(\mathrm{MeO}) \mathrm{C}_{6} \mathrm{H}_{4}$ & 0 \\
$5(\mathrm{e})$ & A & $3,4,5-(\mathrm{MeO})_{3}$ & $4-(\mathrm{MeO}) \mathrm{C}_{6} \mathrm{H}_{4}$ & 92 \\
$6(\mathrm{f})$ & A & $3,4-(\mathrm{MeO})_{2}$ & $4-(\mathrm{MeO}) \mathrm{C}_{6} \mathrm{H}_{4}$ & 90 \\
$7(\mathrm{~g})$ & B & $3,4-(\mathrm{MeO})_{2}$ & $4-(\mathrm{MeO}) \mathrm{C}_{6} \mathrm{H}_{4}$ & 95 \\
\hline
\end{tabular}

Reagents and conditions: (a) $p$ - $\mathrm{TsOH} \cdot \mathrm{H}_{2} \mathrm{O}$ (3.0 equiv), $\mathrm{CH}_{2} \mathrm{Cl}_{2}, 40{ }^{\circ} \mathrm{C}, 8$ h. ${ }^{a}$ Yields refer to chromatographically and spectroscopically homogeneous material.

In addition, we demonstrated a one-pot procedure to prepare hexacyclic benzofuran 20b directly from keto benzyl ether stilbene 17d (Scheme 2). This highly efficient, cascade process involving deprotonation-cyclization (LiTMP), dehydration and Friedel-Crafts ring-closure ( $p$-TsOH) illustrated the utility of the developed methodology in the synthesis of highly functionalized, polycyclic polyphenols, a useful structural class for both chemical and biological investigations.

Scheme 2. One-pot preparation of hexacyclic benzofuran $20 \mathbf{b}$.

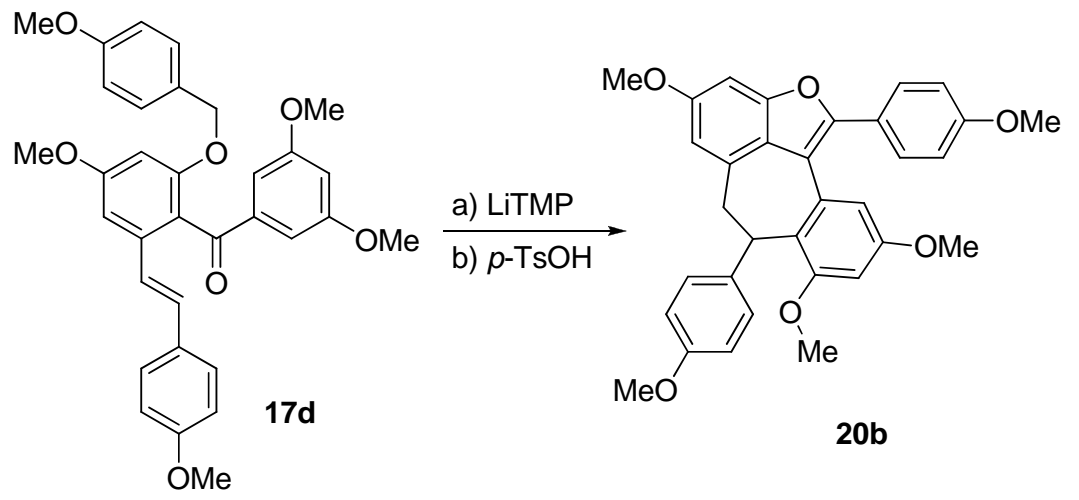

Reagents and conditions: (a) LiTMP (5.0 equiv), THF, $0{ }^{\circ} \mathrm{C}, 0.5 \mathrm{~h}$; (b) $p-\mathrm{TsOH} \cdot \mathrm{H}_{2} \mathrm{O}$ (3.0 equiv), $\mathrm{CH}_{2} \mathrm{Cl}_{2}, 23 \rightarrow 45^{\circ} \mathrm{C}, 8 \mathrm{~h}, 80 \%$ for the two steps. 
Next, the developed methodology was applied to the total synthesis of malibatol A (2) [18] and shoreaphenol (3), as shown in Scheme 3 [19]. In this instance, with pentacyclic benzofuran 19d in hand, construction of the oxygen-substituted, seven-membered ring in the malibatol A (2) and shoreaphenol (3) framework called for an intramolecular Friedel-Crafts type epoxide-opening process. Thus, epoxidation of stilbene 19d under the bromohydrin protocol (NBS, $\mathrm{NaOH}$ ), followed by treatment of the resulting epoxide (21) with $\mathrm{BBr}_{3}$ resulted the concomitant cyclization and global demethylation as a one-pot process, presumably through the intermediacy of 22, giving racemic malibatol A (2) as a single diastereoisomer in 20\% yield. Oxidation of malibatol A (2) in the presence of PDC then afforded shoreaphenol (3), despite the modest yield of $46 \%$. Both malibatol A (2) and shoreaphenol (3) exhibited spectroscopic data $\left({ }^{1} \mathrm{H}-\right.$ and $\left.{ }^{13} \mathrm{C}-\mathrm{NMR}\right)$ and mass spectrometry data matching those reported for the natural substances [11-13].

Scheme 3. Total synthesis of malibatol A (2) and shoreaphenol (3).

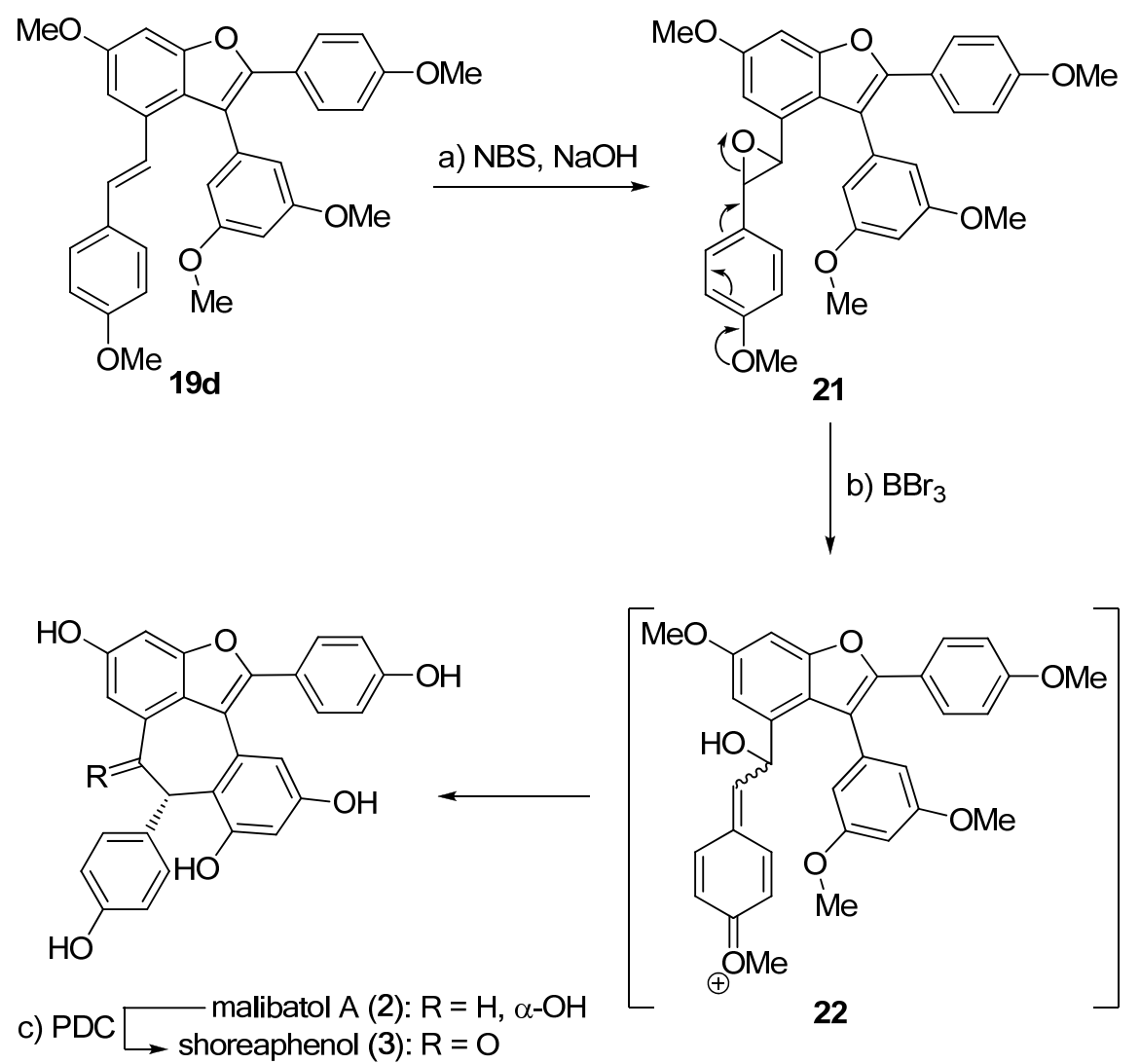

Reagents and conditions: (a) NBS (1.1 equiv), DMSO/ $\mathrm{H}_{2} \mathrm{O}(5: 1), 0^{\circ} \mathrm{C}, 0.5 \mathrm{~h}$; then $\mathrm{NaOH}(4.0 \mathrm{M}$ aq.), $\mathrm{PhEt}_{3} \mathrm{NCl}$ (1.0 equiv), $\mathrm{Et}_{2} \mathrm{O}, 23{ }^{\circ} \mathrm{C}, 2 \mathrm{~h}, 75 \%$; (b) $\mathrm{BBr}_{3}$ (1.0 $\mathrm{M}$ in $\mathrm{CH}_{2} \mathrm{Cl}_{2}, 12$ equiv), $\mathrm{CH}_{2} \mathrm{Cl}_{2}$, $-78 \rightarrow 23{ }^{\circ} \mathrm{C}, 2 \mathrm{~h}, 20 \%$; (c) PDC (1.2 equiv), THF, $0 \rightarrow 23{ }^{\circ} \mathrm{C}, 1 \mathrm{~h}, 46 \%$. NBS $=N$ bromosuccinimide, $\mathrm{DMSO}=$ dimethylsulfoxide, $\mathrm{PDC}=$ pyridinium dichromate.

\section{Experimental}

\subsection{General}

All reactions were carried out under a nitrogen or argon atmosphere with dry solvents under anhydrous conditions, unless otherwise noted. Dry tetrahydrofuran (THF) and methylene chloride 
$\left(\mathrm{CH}_{2} \mathrm{Cl}_{2}\right)$ were obtained by passing commercially available pre-dried, oxygen-free formulations through activated alumina columns. Methanol $(\mathrm{MeOH}), N, N^{\prime}$-dimethylformamide (DMF), dimethylsulfoxide (DMSO) and benzene were purchased in anhydrous form and used without further purification. Acetone, water, ethyl acetate (EtOAc), diethyl ether $\left(\mathrm{Et}_{2} \mathrm{O}\right)$, methylene chloride $\left(\mathrm{CH}_{2} \mathrm{Cl}_{2}\right)$, and hexanes were purchased at the highest commercial quality and used without further purification, unless otherwise stated. Reagents were purchased at the highest commercial quality and used without further purification, unless otherwise stated. Yields refer to chromatographically and spectroscopically ( $\left.{ }^{1} \mathrm{H}-\mathrm{NMR}\right)$ homogeneous materials, unless otherwise stated. Reactions were monitored by thin-layers chromatography (TLC) carried out on $0.25 \mathrm{~mm}$ E. Merck silica gel plates (60F-254) using UV light as visualizing agent and an ethanolic solution of ammonium molybdate and anisaldehyde and heat as developing agents. E. Merck silica gel (60, particle size $0.040-0.063 \mathrm{~mm}$ ) was used for flash column chromatography. ${ }^{1} \mathrm{H}$ and ${ }^{13} \mathrm{C}-\mathrm{NMR}$ spectra were recorded at 600 and $150 \mathrm{MHz}$, respectively, on a Bruker AV-600 instrument and calibrated using residual undeuterated solvent as an internal reference. The following abbreviations were used to explain the multiplicities: $\mathrm{s}=$ singlet, $\mathrm{d}=$ doublet, $\mathrm{t}=$ triplet, $\mathrm{q}=$ quartet, quint $=$ quintet, $\mathrm{m}=$ multiplet, pent $=$ pentet, hex $=$ hexet, $\mathrm{br}=$ broad. IR spectra were recorded on a Perkin-Elmer Spectrum One FTIR spectrometer with diamond ATR accessory. Melting points (m.p.) are uncorrected and were recorded on a Buchi B-540 melting point apparatus. Highresolution mass spectra (HRMS) were recorded on an Agilent ESI TOF (time of flight) mass spectrometer at $3500 \mathrm{~V}$ emitter voltage.

\subsection{General procedure A (Preparation of diaryl ketones 16, Table 1)}

To a solution of aldehyde $15(2.0 \mathrm{mmol})$ in THF $(20 \mathrm{~mL})$ at $0{ }^{\circ} \mathrm{C}$ was added the appropriate Grignard reagent $(0.5 \mathrm{M}$ in THF, $3.0 \mathrm{mmol})$. The resulting mixture was stirred for $0.5 \mathrm{~h}$ before it was quenched with $\mathrm{NH}_{4} \mathrm{Cl}(5 \mathrm{~mL}$, sat. aq.). The layers were separated and the aqueous layer was extracted with EtOAc $(3 \times 10 \mathrm{~mL})$. The combined organic layers were washed with brine $(10 \mathrm{~mL})$, dried $\left(\mathrm{Na}_{2} \mathrm{SO}_{4}\right)$ and concentrated in vacuo to afford the crude benzyl alcohol, which was used directly without further purification. To the solution of crude benzyl alcohol (obtained as above) in DMSO $(5 \mathrm{~mL})$ at $23{ }^{\circ} \mathrm{C}$ was added IBX $(1.15 \mathrm{~g}, 4.1 \mathrm{mmol})$ in one portion. The resulting mixture was stirred for $2 \mathrm{~h}$ before it was quenched with $\mathrm{Na}_{2} \mathrm{~S}_{2} \mathrm{O}_{3}(5 \mathrm{~mL}$, sat. aq.). The layers were separated and the aqueous layer was extracted with $\mathrm{Et}_{2} \mathrm{O}(3 \times 30 \mathrm{~mL})$. The combined organic layers were washed with brine $(30 \mathrm{~mL})$, dried $\left(\mathrm{Na}_{2} \mathrm{SO}_{4}\right)$ and concentrated in vacuo. Flash column chromatography (silica gel) afforded diaryl ketone 16. Using this general procedure the following compounds were prepared:

(2,4-Dimethoxyphenyl)(phenyl)methanone (16a). From 2,4-dimethoxybenzaldehyde and phenylmagnesium bromide. Flash column chromatography (silica gel, hexanes-EtOAc 4:1) afforded ketone 16a (412 mg, 85\%) as a pale yellow foam. All physical properties of this compound were identical to those reported in literature [20].

(E)-(2,4-Dimethoxy-6-(4-methoxystyryl)phenyl)(3,5-dimethoxyphenyl)methanone (16b). From (E)-2,4dimethoxy-6-(4-methoxystyryl)benzaldehyde and 3,5-dimethoxyphenylmagnesium bromide. Flash 
column chromatography (silica gel, hexanes-EtOAc 2:1) afforded ketone 16b (764 mg, 88\%) as a pale yellow foam. All physical properties of this compound were identical to those reported in literature [8].

(E)-(2,4-Dimethoxy-6-(4-methoxystyryl)phenyl)(phenyl)methanone (16f). From (E)-2,4-dimethoxy-6(4-methoxystyryl)benzaldehyde and phenylmagnesium bromide. Flash column chromatography (silica gel, hexanes-EtOAc 2:1) afforded ketone $\mathbf{1 6 f}(636 \mathrm{mg}, 85 \%)$ as a pale yellow foam. $\mathbf{1 6 f}: R_{\mathrm{f}}=0.45$ (silica gel, hexanes-EtOAc 2:1); IR (film) $v_{\max }$ 2938, 1661, 1595, 1510, 1253, 1161, 1078, 920, 830, $721 \mathrm{~cm}^{-1} ;{ }^{1} \mathrm{H}-\mathrm{NMR}\left(\mathrm{CD}_{3} \mathrm{CN}\right): \delta=7.80-7.78(\mathrm{~m}, 2 \mathrm{H}), 7.58-7.55(\mathrm{~m}, 1 \mathrm{H}), 7.46-7.43(\mathrm{~m}, 2 \mathrm{H}), 7.25$ $(\mathrm{d}, J=9.0 \mathrm{~Hz}, 2 \mathrm{H}), 7.13(\mathrm{~d}, J=16.2 \mathrm{~Hz}, 1 \mathrm{H}), 6.97(\mathrm{~d}, J=1.8 \mathrm{~Hz}, 1 \mathrm{H}), 6.81(\mathrm{~d}, J=9.0 \mathrm{~Hz}, 2 \mathrm{H})$, $6.70(\mathrm{~d}, J=16.2 \mathrm{~Hz}, 1 \mathrm{H}), 6.56(\mathrm{~d}, J=1.8 \mathrm{~Hz}, 1 \mathrm{H}), 3.89(\mathrm{~s}, 3 \mathrm{H}), 3.71(\mathrm{~s}, 3 \mathrm{H}), 3.63 \mathrm{ppm}(\mathrm{s}, 3 \mathrm{H})$; ${ }^{13} \mathrm{C}-\mathrm{NMR}\left(\mathrm{CD}_{3} \mathrm{OD}\right): \delta=197.2,161.6,159.7,158.3,138.3,137.4,133.5,131.2,129.4,129.1,128.7$, 127.9, 122.4, 121.0, 114.1, 101.4, 97.7, 55.5, 55.3, 54.9 ppm; HRMS (ESI): calcd for $\mathrm{C}_{24} \mathrm{H}_{22} \mathrm{O}_{4} \mathrm{Na}^{+}$[M $+\mathrm{Na}^{+}$] 397.1410, found 397.1406.

(E)-(2,4-Dimethoxy-6-(4-methoxystyryl)phenyl)(3,4,5-trimethoxyphenyl)methanone (16g). From (E)2,4-dimethoxy-6-(4-methoxystyryl)benzaldehyde and 3,4,5-trimethoxyphenylmagnesium bromide. Flash column chromatography (silica gel, hexanes-EtOAc 2:1) afforded ketone $\mathbf{1 6 g}(836 \mathrm{mg}, 90 \%)$ as a pale yellow foam. 16g: $R_{\mathrm{f}}=0.25$ (silica gel, hexanes-EtOAc 2:1); IR (film) $v_{\max } 2938,1661,1578$, 1511, 1413, 1327, 1156, 1126, $834 \mathrm{~cm}^{-1}$; ${ }^{1} \mathrm{H}-\mathrm{NMR}\left(\mathrm{CD}_{3} \mathrm{CN}\right): \delta=7.28(\mathrm{~d}, J=8.4 \mathrm{~Hz}, 2 \mathrm{H}), 7.13(\mathrm{~d}$, $J=16.2 \mathrm{~Hz}, 1 \mathrm{H}), 7.08(\mathrm{~s}, 2 \mathrm{H}), 6.95(\mathrm{~d}, J=2.4 \mathrm{~Hz}, 1 \mathrm{H}), 6.84(\mathrm{~d}, J=8.4 \mathrm{~Hz}, 2 \mathrm{H}), 6.67(\mathrm{~d}$, $J=16.2 \mathrm{~Hz}, 1 \mathrm{H}), 6.56(\mathrm{~d}, J=2.4 \mathrm{~Hz}, 1 \mathrm{H}), 3.89$ (s, $3 \mathrm{H}), 3.77(\mathrm{~s}, 3 \mathrm{H}), 3.75(\mathrm{~s}, 6 \mathrm{H}), 3.74(\mathrm{~s}, 3 \mathrm{H})$, $3.68 \mathrm{ppm}(\mathrm{s}, 3 \mathrm{H}) ;{ }^{13} \mathrm{C}-\mathrm{NMR}\left(\mathrm{CD}_{3} \mathrm{OD}\right): \delta=195.8,161.5,159.7,158.2,153.3,142.7,137.5,133.6$, 131.1, 129.4, 127.9, 122.6, 120.8, 114.1, 106.6, 101.4, 97.7, 60.0, 55.7, 55.5, 55.3, 54.9 ppm; HRMS (ESI): calcd for $\mathrm{C}_{27} \mathrm{H}_{28} \mathrm{O}_{7} \mathrm{Na}^{+}\left[\mathrm{M}+\mathrm{Na}^{+}\right]$487.1727, found 487.1712.

(E)-(2,4-dimethoxy-6-(4-methoxystyryl)phenyl)(3,4-dimethoxyphenyl)methanone (16h). From (E)-2,4dimethoxy-6-(4-methoxystyryl)benzaldehyde and 3,4-dimethoxyphenylmagnesium bromide. Flash column chromatography (silica gel, hexanes-EtOAc 2:1) afforded ketone 16h (755 $\mathrm{mg}, 87 \%$ ) as a pale yellow foam. 16h: $R_{\mathrm{f}}=0.20$ (silica gel, hexanes-EtOAc 2:1); IR (film) $v_{\max } 2937,1739,1653,1595$, 1511, 1267, 1158, $835 \mathrm{~cm}^{-1}$; ${ }^{1} \mathrm{H}-\mathrm{NMR}\left(\mathrm{CD}_{3} \mathrm{CN}\right): \delta=7.50(\mathrm{~d}, J=1.8 \mathrm{~Hz}, 1 \mathrm{H}), 7.26(\mathrm{~d}, J=9.0 \mathrm{~Hz}$, $2 \mathrm{H}), 7.20(\mathrm{dd}, J=8.4,2.4 \mathrm{~Hz}, 1 \mathrm{H}), 7.13(\mathrm{~d}, J=16.2 \mathrm{~Hz}, 1 \mathrm{H}), 6.94(\mathrm{~d}, J=2.4 \mathrm{~Hz}, 1 \mathrm{H}), 6.90(\mathrm{~d}$, $J=8.4 \mathrm{~Hz}, 1 \mathrm{H}), 6.83(\mathrm{~d}, J=9.0 \mathrm{~Hz}, 2 \mathrm{H}), 6.65(\mathrm{~d}, J=16.2 \mathrm{~Hz}, 1 \mathrm{H}), 6.55(\mathrm{~d}, J=1.8 \mathrm{~Hz}, 1 \mathrm{H}), 3.89$ $(\mathrm{s}, 3 \mathrm{H}), 3.84(\mathrm{~s}, 3 \mathrm{H}), 3.83(\mathrm{~s}, 3 \mathrm{H}), 3.74(\mathrm{~s}, 3 \mathrm{H}), 3.67 \mathrm{ppm}(\mathrm{s}, 3 \mathrm{H}) ;{ }^{13} \mathrm{C}-\mathrm{NMR}\left(\mathrm{CD}_{3} \mathrm{CN}\right): \delta=195.5$, $161.3,159.7,158.0,153.8,149.2,137.1,131.3,130.9,129.4,127.8,125.1,122.5,121.3,114.1,110.5$, 110.1, 101.1, 97.7, 55.5, 55.5, 55.3, 55.2, 54.9 ppm; HRMS (ESI): calcd for $\mathrm{C}_{26} \mathrm{H}_{26} \mathrm{O}_{6} \mathrm{Na}^{+}\left[\mathrm{M}+\mathrm{Na}^{+}\right]$ 457.1621 , found 457.1610 .

(2,4-Dimethoxy-6-((4-methoxyphenyl)ethynyl)phenyl)(3,5-dimethoxyphenyl)methanone (16i). From 2,4-dimethoxy-6-[(4-methoxyphenyl)ethynyl]benzaldehyde and 3,5-dimethoxyphenylmagnesium bromide. Flash column chromatography (silica gel, hexanes-EtOAc 2:1) afforded ketone 16i (743 mg, 86\%) as a pale yellow foam. 16i: $R_{\mathrm{f}}=0.28$ (silica gel, hexanes-EtOAc 2:1); IR (film) $v_{\max } 2938,1671$, 1590, 1569, 1510, 1247, 1153, 1065, $832 \mathrm{~cm}^{-1} ;{ }^{1} \mathrm{H}-\mathrm{NMR}\left(\mathrm{CD}_{3} \mathrm{CN}\right): \delta=7.10(\mathrm{~d}, J=9.0 \mathrm{~Hz}, 2 \mathrm{H}), 6.92$ 
$(\mathrm{d}, J=2.4 \mathrm{~Hz}, 2 \mathrm{H}), 6.84(\mathrm{~d}, J=9.0 \mathrm{~Hz}, 2 \mathrm{H}), 6.73(\mathrm{~d}, J=2.4 \mathrm{~Hz}, 1 \mathrm{H}), 6.72(\mathrm{t}, J=2.4 \mathrm{~Hz}, 1 \mathrm{H}), 6.66$ $(\mathrm{d}, J=2.4 \mathrm{~Hz}, 1 \mathrm{H}), 3.86(\mathrm{~s}, 3 \mathrm{H}), 3.77(\mathrm{~s}, 6 \mathrm{H}), 3.75(\mathrm{~s}, 3 \mathrm{H}), 3.72 \mathrm{ppm}(\mathrm{s}, 3 \mathrm{H}) ;{ }^{13} \mathrm{C}-\mathrm{NMR}\left(\mathrm{CD}_{3} \mathrm{CN}\right)$ : $\delta=195.7,162.3,162.0,161.0,158.8,140.7,133.6,125.0,123.2,115.0,114.8,108.6,107.7,106.1$, 100.3, 94.2, 86.4, 56.5, 56.3, 56.2, 55.9 ppm; HRMS (ESI): calcd for $\mathrm{C}_{26} \mathrm{H}_{24} \mathrm{O}_{6} \mathrm{Na}^{+}\left[\mathrm{M}+\mathrm{Na}^{+}\right]$ 455.1465 , found 455.1467 .

\subsection{General procedure B (Preparation of benzyl ethers 17, Table 1)}

To a solution of diaryl ketone $16(1.0 \mathrm{mmol})$ in $\mathrm{CH}_{2} \mathrm{Cl}_{2}(10 \mathrm{~mL})$ at $0{ }^{\circ} \mathrm{C}$ was added $\mathrm{BCl}_{3}(1.0 \mathrm{M}$ in $\left.\mathrm{CH}_{2} \mathrm{Cl}_{2}, 1.5 \mathrm{~mL}, 1.5 \mathrm{mmol}\right)$ dropwise. The resulting mixture was stirred for $1 \mathrm{~h}$ before it was quenched with $\mathrm{NH}_{4} \mathrm{Cl}(10 \mathrm{~mL}$, sat. aq.). The layers were separated and the aqueous layer was extracted with $\mathrm{CH}_{2} \mathrm{Cl}_{2}(3 \times 10 \mathrm{~mL})$. The combined organic layers were washed with brine $(20 \mathrm{~mL})$, dried $\left(\mathrm{Na}_{2} \mathrm{SO}_{4}\right)$ and concentrated in vacuo to afford the crude phenol, which was used directly without further purification. To a solution of the crude phenol (obtained as above) in DMF $(5 \mathrm{~mL})$ at $0{ }^{\circ} \mathrm{C}$ was added $\mathrm{NaH}(80 \mathrm{mg}, 60 \% \mathrm{wt} / \mathrm{wt}$ in mineral oil, $2.0 \mathrm{mmol})$. The resulting mixture was stirred for $0.5 \mathrm{~h}$ before benzyl bromide (or chloride) $\left(1.4 \mathrm{mmol}\right.$ ) was added. The reaction mixture was warmed to $23{ }^{\circ} \mathrm{C}$ and the progress was monitored by TLC analysis. Upon completion of the reaction ( $<4 \mathrm{~h}$ for most cases), the reaction mixture was quenched with $\mathrm{NH}_{4} \mathrm{Cl}(20 \mathrm{~mL}$, sat. aq.). The layers were separated and the aqueous layer was extracted with $\mathrm{Et}_{2} \mathrm{O}(3 \times 20 \mathrm{~mL})$. The combined organic layers were washed with brine $(30 \mathrm{~mL})$, dried $\left(\mathrm{Na}_{2} \mathrm{SO}_{4}\right)$ and concentrated in vacuo. Flash column chromatography (silica gel) afforded the desired benzyl ether 17. Using the described general procedure the following substances were prepared:

(2-(Benzyloxy)-4-methoxyphenyl)(phenyl)methanone (17a). From ketone 16a and benzyl bromide. Flash column chromatography (silica gel, hexanes-EtOAc 4:1) afforded benzyl ether 17a (283 mg, 89\%) as a yellow foam. 17a: $R_{\mathrm{f}}=0.35$ (silica gel, hexanes-EtOAc 4:1); IR (film) $v_{\max } 1651,1601$, 1579, 1501, 1446, 1272, 1166, 1120, 737, $697 \mathrm{~cm}^{-1} ;{ }^{1} \mathrm{H}-\mathrm{NMR}\left(\mathrm{CD}_{3} \mathrm{CN}\right): \delta=7.73-7.72(\mathrm{~m}, 2 \mathrm{H})$, 7.57-7.55 (m, 1 H), 7.46-7.41 (m, 3 H), 7.21-7.16 (m, 3 H), 6.93-6.92 (m, $2 \mathrm{H}), 6.69$ (d, J = 2.4 Hz, $1 \mathrm{H}), 6.64(\mathrm{dd}, J=8.4,2.4 \mathrm{~Hz}, 1 \mathrm{H}), 4.97(\mathrm{~s}, 2 \mathrm{H}), 3.84 \mathrm{ppm}(\mathrm{s}, 3 \mathrm{H}) ;{ }^{13} \mathrm{C}-\mathrm{NMR}\left(\mathrm{CD}_{3} \mathrm{CN}\right): \delta=195.6$, 163.4, 158.3, 139.2, 136.5, 132.5, 131.6, 129.2, 128.3, 128.2, 127.6, 126.9, 121.7, 105.6, 99.7, 69.8, 55.4 ppm; HRMS (ESI): calcd for $\mathrm{C}_{21} \mathrm{H}_{18} \mathrm{O}_{3} \mathrm{Na}^{+}\left[\mathrm{M}+\mathrm{Na}^{+}\right] 341.1148$, found 341.1156.

(E)-(2-(Benzyloxy)-4-methoxy-6-(4-methoxystyryl)phenyl)(3,5-dimethoxyphenyl)methanone

(17b).

From ketone 16b and benzyl bromide. Flash column chromatography (silica gel, hexanes-EtOAc 2:1) afforded benzyl ether 17b (485 mg, 95\%) as a yellow foam. 17b: $R_{\mathrm{f}}=0.45$ (silica gel, hexanes-EtOAc 2:1); IR (film) $v_{\max } 1667,1594,1511,1301,1204,1156,1065,829 \mathrm{~cm}^{-1} ;{ }^{1} \mathrm{H}-\mathrm{NMR}\left(\mathrm{CD}_{3} \mathrm{CN}\right): \delta=7.31$ $(\mathrm{d}, J=9.0 \mathrm{~Hz}, 2 \mathrm{H}), 7.24-7.23(\mathrm{~m}, 3 \mathrm{H}), 7.14(\mathrm{~d}, J=16.2 \mathrm{~Hz}, 1 \mathrm{H}), 7.04-7.02(\mathrm{~m}, 2 \mathrm{H}), 6.96(\mathrm{~d}$, $J=2.4 \mathrm{~Hz}, 1 \mathrm{H}), 6.88(\mathrm{~d}, J=2.4 \mathrm{~Hz}, 2 \mathrm{H}), 6.86(\mathrm{~d}, J=9.0 \mathrm{~Hz}, 2 \mathrm{H}), 6.73-6.70(\mathrm{~m}, 2 \mathrm{H}), 6.59(\mathrm{~d}$, $J=1.8 \mathrm{~Hz}, 1 \mathrm{H}), 4.99(\mathrm{~s}, 2 \mathrm{H}), 3.88(\mathrm{~s}, 3 \mathrm{H}), 3.76(\mathrm{~s}, 3 \mathrm{H}), 3.75 \mathrm{ppm}(\mathrm{s}, 6 \mathrm{H}) ;{ }^{13} \mathrm{C}-\mathrm{NMR}\left(\mathrm{CD}_{3} \mathrm{CN}\right)$ : $\delta=196.8,161.5,161.1,159.8,157.2,140.8,137.7,136.6,131.3,129.4,128.3,127.9,127.8,127.2$, 122.4, 121.3, 114.1, 106.7, 105.1, 101.7, 99.0, 70.0, 55.3, 55.3, 54.9 ppm; HRMS (ESI): calcd for $\mathrm{C}_{32} \mathrm{H}_{30} \mathrm{O}_{6} \mathrm{Na}^{+}\left[\mathrm{M}+\mathrm{Na}^{+}\right]$533.1934, found 533.1951. 
(E)-(2-((4-Bromobenzyl)oxy)-4-methoxy-6-(4-methoxystyryl)phenyl)(3,5-dimethoxyphenyl)methanone (17c). From ketone 16b and 4-bromobenzyl bromide. Flash column chromatography (silica gel, hexanes-EtOAc 2:1) afforded benzyl ether $17 \mathrm{c}(542 \mathrm{mg}, 92 \%)$ as a yellow foam. 17c: $R_{\mathrm{f}}=0.41$ (silica gel, hexanes-EtOAc 2:1); IR (film) $v_{\max } 2837,1666,1593,1510,1300,1156,1066,806 \mathrm{~cm}^{-1} ;{ }^{1} \mathrm{H}-$ NMR $\left(\mathrm{CD}_{3} \mathrm{CN}\right): \delta=7.37(\mathrm{~d}, J=8.4 \mathrm{~Hz}, 2 \mathrm{H}), 7.31(\mathrm{~d}, J=9.0 \mathrm{~Hz}, 2 \mathrm{H}), 7.15(\mathrm{~d}, J=16.2 \mathrm{~Hz}, 1 \mathrm{H})$, $6.97(\mathrm{~d}, J=2.4 \mathrm{~Hz}, 1 \mathrm{H}), 6.93(\mathrm{~d}, J=8.4 \mathrm{~Hz}, 2 \mathrm{H}), 6.86$ (d, $J=9.0 \mathrm{~Hz}, 2 \mathrm{H}), 6.84(\mathrm{~s}, 2 \mathrm{H}), 6.72$ (d, $J=16.2 \mathrm{~Hz}, 1 \mathrm{H}), 6.70(\mathrm{t}, J=2.4 \mathrm{~Hz}, 1 \mathrm{H}), 6.58(\mathrm{~d}, J=2.4 \mathrm{~Hz}, 1 \mathrm{H}), 4.95$ (s, $2 \mathrm{H}), 3.88$ (s, $3 \mathrm{H}), 3.75$ $(\mathrm{s}, 3 \mathrm{H}), 3.74 \mathrm{ppm}(\mathrm{s}, 6 \mathrm{H}) ;{ }^{13} \mathrm{C}-\mathrm{NMR}\left(\mathrm{CD}_{3} \mathrm{CN}\right): \delta=196.8,161.5,161.1,159.8,157.1,140.9,137.9$, 135.9, 131.3, 131.3, 129.4, 129.1, 127.9, 122.3, 121.3, 121.1, 114.1, 106.6, 105.1, 101.9, 99.0, 69.3, 55.3, 55.3, 54.9 ppm; HRMS (ESI): calcd for $\mathrm{C}_{32} \mathrm{H}_{29} \mathrm{BrO}_{6} \mathrm{Na}^{+}\left[\mathrm{M}+\mathrm{Na}^{+}\right]$611.1039, found 611.1033.

(E)-(3,5-Dimethoxyphenyl)(4-methoxy-2-((4-methoxybenzyl)oxy)-6-(4-methoxystyryl)phenyl)-methanone (17d). From ketone 16b and 4-methoxybenzyl chloride. Flash column chromatography (silica gel, hexanes-EtOAc 4:1) afforded benzyl ether $\mathbf{1 7 d}(486 \mathrm{mg}, 90 \%)$ as a pale yellow solid. $\mathbf{1 7 d}$ : $R_{\mathrm{f}}=0.40$ (silica gel, hexanes-EtOAc 2:1); m.p. $=118-119{ }^{\circ} \mathrm{C}$ (hexanes-EtOAc); IR (film) $v_{\max } 2970,1738$, 1594, 1512, 1352, 1302, 1249, 1204, 1156, 1065, $834 \mathrm{~cm}^{-1} ;{ }^{1} \mathrm{H}-\mathrm{NMR}\left(\mathrm{CDCl}_{3}\right): \delta=7.31(\mathrm{~d}, J=9.0 \mathrm{~Hz}$, $2 \mathrm{H}), 7.03(\mathrm{~d}, J=15.0 \mathrm{~Hz}, 1 \mathrm{H}), 6.98(\mathrm{~d}, J=2.4 \mathrm{~Hz}, 2 \mathrm{H}), 6.92(\mathrm{~d}, J=9.0 \mathrm{~Hz}, 2 \mathrm{H}), 6.86(\mathrm{~d}, J=2.4$ $\mathrm{Hz}, 1 \mathrm{H}), 6.85(\mathrm{~d}, J=14.4 \mathrm{~Hz}, 1 \mathrm{H}), 6.82(\mathrm{~d}, J=9.0 \mathrm{~Hz}, 2 \mathrm{H}), 6.74(\mathrm{~d}, J=9.0 \mathrm{~Hz}, 2 \mathrm{H}), 6.66(\mathrm{t}, J=2.4$ $\mathrm{Hz}, 1 \mathrm{H}), 6.45(\mathrm{~d}, J=2.4 \mathrm{~Hz}, 1 \mathrm{H}), 4.88(\mathrm{~s}, 2 \mathrm{H}), 3.87(\mathrm{~s}, 3 \mathrm{H}), 3.77(\mathrm{~s}, 6 \mathrm{H}), 3.76(\mathrm{~s}, 3 \mathrm{H}), 3.75 \mathrm{ppm}$ $(\mathrm{s}, 3 \mathrm{H}) ;{ }^{13} \mathrm{C}-\mathrm{NMR}\left(\mathrm{CDCl}_{3}\right): \delta=197.3,161.1,160.6,159.3,158.9,157.3,140.9,137.8,130.8,129.4$, 128.4, 128.2, 127.9, 122.8, 121.6, 113.8, 113.4, 106.9, 105.3, 101.2, 98.9, 69.8, 55.4, 55.3, 55.1, 55.0 ppm; HRMS (ESI): calcd for $\mathrm{C}_{33} \mathrm{H}_{32} \mathrm{O}_{7} \mathrm{Na}^{+}\left[\mathrm{M}+\mathrm{Na}^{+}\right]$563.2040, found 563.2037.

\section{(E)-(3,5-Dimethoxyphenyl)(2-(furan-2-ylmethoxy)-4-methoxy-6-(4-methoxystyryl)phenyl)methanone}

(17e). To a solution of phenol $\mathbf{1 6 b}(420 \mathrm{mg}, 1.0 \mathrm{mmol})$ in THF $(10 \mathrm{~mL})$ at $23{ }^{\circ} \mathrm{C}$ was added $\mathrm{PPh}_{3}$ (786 mg, $3.0 \mathrm{mmol})$. The resulting mixture was cooled to $0{ }^{\circ} \mathrm{C}$ before a solution of DEAD (522 $\mathrm{mg}$, $3.0 \mathrm{mmol})$ and furfuryl alcohol $(294 \mathrm{mg}, 3.0 \mathrm{mmol})$ in THF $(2 \mathrm{~mL})$ were added. The resulting mixture was warmed to $23{ }^{\circ} \mathrm{C}$ and stirred for $12 \mathrm{~h}$ before it was quenched with $\mathrm{NH}_{4} \mathrm{Cl}$ (5 mL, sat. aq.). The layers were separated and the aqueous layer was extracted with EtOAc $(3 \times 10 \mathrm{~mL})$. The combined organic layers were washed with brine $(10 \mathrm{~mL})$, dried $\left(\mathrm{Na}_{2} \mathrm{SO}_{4}\right)$ and concentrated in vacuo. Flash column chromatography (silica gel, hexanes-EtOAc 4:1) afforded furanyl ether 17e (355 mg, 71\%) as a yellow oil. 17e: $R_{\mathrm{f}}=0.72$ (silica gel, benzene-EtOAc 8:1); IR (film) $v_{\max } 2937,1667,1592,1510$, 1456, 1300, 1155, 1063, $819 \mathrm{~cm}^{-1} ;{ }^{1} \mathrm{H}-\mathrm{NMR}\left(\mathrm{CD}_{3} \mathrm{CN}\right): \delta=7.38(\mathrm{~d}, J=1.2 \mathrm{~Hz}, 1 \mathrm{H}), 7.28(\mathrm{~d}$, $J=9.0 \mathrm{~Hz}, 2 \mathrm{H}), 7.13(\mathrm{~d}, J=16.2 \mathrm{~Hz}, 1 \mathrm{H}), 6.97(\mathrm{~d}, J=2.4 \mathrm{~Hz}, 1 \mathrm{H}), 6.85(\mathrm{~d}, J=9.0 \mathrm{~Hz}, 2 \mathrm{H}), 6.82$ $(\mathrm{d}, J=2.4 \mathrm{~Hz}, 2 \mathrm{H}), 6.69(\mathrm{t}, J=2.4 \mathrm{~Hz}, 1 \mathrm{H}), 6.68(\mathrm{~d}, J=2.4 \mathrm{~Hz}, 1 \mathrm{H}), 6.65$ (t, $J=16.2 \mathrm{~Hz}, 1 \mathrm{H})$, 6.33-6.32 (m, $1 \mathrm{H}), 6.28(\mathrm{~d}, J=3.6 \mathrm{~Hz}, 1 \mathrm{H}), 4.95(\mathrm{~s}, 2 \mathrm{H}), 3.90$ (s, $3 \mathrm{H}), 3.75(\mathrm{~s}, 3 \mathrm{H}), 3.74 \mathrm{ppm}(\mathrm{s}$, $6 \mathrm{H}) ;{ }^{13} \mathrm{C}-\mathrm{NMR}\left(\mathrm{CD}_{3} \mathrm{CN}\right): \delta=197.4,162.2,161.9,160.6,157.6,150.7,144.2,141.3,138.5,132.2$, 130.2, 128.8, 123.1, 122.3, 115.0, 111.3, 111.1, 107.6, 106.0, 103.0, 100.1, 63.5, 56.2, 56.1, 55.8 ppm; HRMS (ESI): calcd for $\mathrm{C}_{30} \mathrm{H}_{28} \mathrm{O}_{7} \mathrm{Na}^{+}\left[\mathrm{M}+\mathrm{Na}^{+}\right]$523.1727, found 523.1725.

(E)-(4-Methoxy-2-((4-methoxybenzyl)oxy)-6-(4-methoxystyryl)phenyl)(phenyl)methanone (17f). From ketone 16f and 4-methoxybenzyl chloride. Flash column chromatography (silica gel, hexanes-EtOAc 
2:1) afforded benzyl ether 17f (437 $\mathrm{mg}, 91 \%)$ as a yellow foam. 17f: $R_{\mathrm{f}}=0.50$ (silica gel, hexanesEtOAc 2:1); IR (film) $v_{\max } 1661,1595,1511,1249,1163,1033,827,721 \mathrm{~cm}^{-1} ;{ }^{1} \mathrm{H}-\mathrm{NMR}\left(\mathrm{CD}_{3} \mathrm{CN}\right): \delta$ $=7.77-7.75(\mathrm{~m}, 2 \mathrm{H}), 7.62-7.59(\mathrm{~m}, 1 \mathrm{H}), 7.47(\mathrm{t}, J=7.8 \mathrm{~Hz}, 2 \mathrm{H}), 7.28(\mathrm{~d}, J=9.0 \mathrm{~Hz}, 2 \mathrm{H}), 7.14(\mathrm{~d}$, $J=16.2 \mathrm{~Hz}, 1 \mathrm{H}), 6.97(\mathrm{~d}, J=1.8 \mathrm{~Hz}, 1 \mathrm{H}), 6.89$ (d, $J=8.4 \mathrm{~Hz}, 2 \mathrm{H}), 6.83(\mathrm{~d}, J=9.0 \mathrm{~Hz}, 2 \mathrm{H}), 6.74$ $(\mathrm{d}, J=8.4 \mathrm{~Hz}, 2 \mathrm{H}), 6.72(\mathrm{~d}, J=16.2 \mathrm{~Hz}, 1 \mathrm{H}), 6.61(\mathrm{~d}, J=1.8 \mathrm{~Hz}, 1 \mathrm{H}), 4.89$ (s, $2 \mathrm{H}), 3.89(\mathrm{~s}, 3 \mathrm{H})$, $3.74(\mathrm{~s}, 3 \mathrm{H}), 3.71 \mathrm{ppm}(\mathrm{s}, 3 \mathrm{H}) ;{ }^{13} \mathrm{C}-\mathrm{NMR}\left(\mathrm{CD}_{3} \mathrm{CN}\right): \delta=198.5,162.8,161.0,160.6,158.7,139.9$, 139.0, 134.7, 132.5, 130.7, 130.4, 130.3, 130.0, 129.7, 129.2, 123.7, 122.8, 115.4, 114.9, 103.0, 100.4, 71.2, 56.6, 56.2, 56.1 ppm; HRMS (ESI): calcd for $\mathrm{C}_{31} \mathrm{H}_{28} \mathrm{O}_{5} \mathrm{Na}^{+}\left[\mathrm{M}+\mathrm{Na}^{+}\right] 503.1829$, found 503.1817 .

(E)-(4-Methoxy-2-((4-methoxybenzyl)oxy)-6-(4-methoxystyryl)phenyl)(3,4,5-trimethoxyphenyl)methanone $(\mathbf{1 7 g})$. From ketone 16g and 4-methoxybenzyl chloride. Flash column chromatography (silica gel, hexanes-EtOAc 2:1) afforded benzyl ether $\mathbf{1 7 g}\left(542 \mathrm{mg}, 95 \%\right.$ ) as a yellow foam. $\mathbf{1 7 g}: R_{\mathrm{f}}=0.30$ (silica gel, hexanes-EtOAc 2:1); IR (film) $v_{\max } 1655,1595,1512,1413,1327,1249,1157,1126,832 \mathrm{~cm}^{-1}$; ${ }^{1} \mathrm{H}-\mathrm{NMR}\left(d_{6}\right.$-acetone): $\delta 7.35(\mathrm{~d}, J=9.0 \mathrm{~Hz}, 2 \mathrm{H}), 7.22(\mathrm{~d}, J=15.6 \mathrm{~Hz}, 1 \mathrm{H}), 7.08(\mathrm{~s}, 2 \mathrm{H}), 7.05(\mathrm{~d}$, $J=2.4 \mathrm{~Hz}, 1 \mathrm{H}), 6.97$ (d, $J=8.4 \mathrm{~Hz}, 2 \mathrm{H}), 6.88(\mathrm{~d}, J=8.4 \mathrm{~Hz}, 2 \mathrm{H}), 6.85(\mathrm{~d}, J=15.6 \mathrm{~Hz}, 1 \mathrm{H}), 6.77$ $(\mathrm{d}, J=8.4 \mathrm{~Hz}, 2 \mathrm{H}), 6.68(\mathrm{~d}, J=2.4 \mathrm{~Hz}, 1 \mathrm{H}), 4.99(\mathrm{~s}, 2 \mathrm{H}), 3.91(\mathrm{~s}, 3 \mathrm{H}), 3.81(\mathrm{~s}, 3 \mathrm{H}), 3.77(\mathrm{~s}, 9 \mathrm{H})$, 3.74 ppm (s, $3 \mathrm{H}) ;{ }^{13} \mathrm{C}-\mathrm{NMR}\left(d_{6}\right.$-acetone): $\delta=195.3,161.4,159.8,159.3,157.5,153.4,142.8,137.7$, 134.4, 130.8, 129.6, 128.7, 128.6, 127.9, 122.7, 121.6, 114.0, 113.4, 106.6, 101.4, 99.0, 69.6, 59.8, 55.6, 54.9, 54.6, 54.5 ppm; HRMS (ESI): calcd for $\mathrm{C}_{34} \mathrm{H}_{34} \mathrm{O}_{8} \mathrm{Na}^{+}\left[\mathrm{M}+\mathrm{Na}^{+}\right] 593.2145$, found 593.2137.

(E)-(3,4-Dimethoxyphenyl)(4-methoxy-2-((4-methoxybenzyl)oxy)-6-(4-methoxystyryl)phenyl)methanone (17h). From ketone 16h and 4-methoxybenzyl chloride. Flash column chromatography (silica gel, hexanes-EtOAc 2:1) afforded benzyl ether $\mathbf{1 7 h}(486 \mathrm{mg}, 90 \%)$ as a yellow foam. 17h: $R_{\mathrm{f}}=0.25$ (silica gel, hexanes-EtOAc 2:1); IR (film) $v_{\max } 1652,1594,1510,1265,1249,1159,1121,1024,833 \mathrm{~cm}^{-1}$; ${ }^{1} \mathrm{H}-\mathrm{NMR}\left(\mathrm{CD}_{3} \mathrm{CN}\right): \delta 7.45(\mathrm{~d}, J=1.8 \mathrm{~Hz}, 1 \mathrm{H}), 7.27(\mathrm{~d}, J=9.0 \mathrm{~Hz}, 2 \mathrm{H}), 7.20(\mathrm{dd}, J=8.4,1.8 \mathrm{~Hz}, 1$ H), $7.13(\mathrm{~d}, J=16.2 \mathrm{~Hz}, 1 \mathrm{H}), 6.98(\mathrm{~d}, J=8.4 \mathrm{~Hz}, 2 \mathrm{H}), 6.95(\mathrm{~d}, J=2.4 \mathrm{~Hz}, 1 \mathrm{H}), 6.89$ (d, $J=8.4 \mathrm{~Hz}$, $1 \mathrm{H}), 6.83(\mathrm{~d}, J=9.0 \mathrm{~Hz}, 2 \mathrm{H}), 6.76(\mathrm{~d}, J=9.0 \mathrm{~Hz}, 2 \mathrm{H}), 6.70(\mathrm{~d}, J=16.2 \mathrm{~Hz}, 1 \mathrm{H}), 6.60(\mathrm{~d}, J=2.4$ $\mathrm{Hz}, 1 \mathrm{H}), 4.91$ (s, $2 \mathrm{H}), 3.87$ (s, $3 \mathrm{H}), 3.83$ (s, $3 \mathrm{H}), 3.80$ (s, $3 \mathrm{H}), 3.73(\mathrm{~s}, 3 \mathrm{H}), 3.72 \mathrm{ppm}(\mathrm{s}, 3 \mathrm{H}) ;{ }^{13} \mathrm{C}-$ NMR $\left(\mathrm{CD}_{3} \mathrm{CN}\right): \delta=195.7,161.2,159.7,159.3,157.1,153.8,149.2,137.3,131.6,130.9,129.4,129.0$, 128.5, 127.8, 124.9, 122.5, 121.9, 114.1, 113.6, 110.5, 110.2, 101.4, 99.2, 69.9, 55.6, 55.3, 55.3, 54.9, 54.8 ppm; HRMS (ESI): calcd for $\mathrm{C}_{33} \mathrm{H}_{32} \mathrm{O}_{7} \mathrm{Na}^{+}\left[\mathrm{M}+\mathrm{Na}^{+}\right]$563.2040, found 563.2057.

\section{(3,5-Dimethoxyphenyl)(4-methoxy-2-((4-methoxybenzyl)oxy)-6-((4-methoxyphenyl)ethynyl)phenyl)}

methanone (17i). From ketone 16i and 4-methoxybenzyl chloride. Flash column chromatography (silica gel, hexanes-EtOAc 2:1) afforded benzyl ether 17i (468 $\mathrm{mg}, 87 \%$ ) as a yellow foam. 17i: $R_{\mathrm{f}}=0.27$ (silica gel, hexanes-EtOAc 2:1); IR (film) $v_{\max } 2937,1671,1591,1511,1300,1248,1155$, 1063, $832 \mathrm{~cm}^{-1}$; ${ }^{1} \mathrm{H}-\mathrm{NMR}\left(\mathrm{CD}_{3} \mathrm{CN}\right): \delta=7.12(\mathrm{~d}, J=8.4 \mathrm{~Hz}, 2 \mathrm{H}), 7.07(\mathrm{~d}, J=9.0 \mathrm{~Hz}, 2 \mathrm{H}), 6.88(\mathrm{~d}$, $J=2.4 \mathrm{~Hz}, 2 \mathrm{H}), 6.84(\mathrm{~d}, J=9.0 \mathrm{~Hz}, 2 \mathrm{H}), 6.81(\mathrm{~d}, J=8.4 \mathrm{~Hz}, 2 \mathrm{H}), 6.74(\mathrm{~d}, J=2.4 \mathrm{~Hz}, 1 \mathrm{H}), 6.72(\mathrm{t}$, $J=2.4 \mathrm{~Hz}, 1 \mathrm{H}), 6.71(\mathrm{~d}, J=2.4 \mathrm{~Hz}, 1 \mathrm{H}), 4.97(\mathrm{~s}, 2 \mathrm{H}), 3.85(\mathrm{~s}, 3 \mathrm{H}), 3.77(\mathrm{~s}, 6 \mathrm{H}), 3.76$ (s, $3 \mathrm{H}), 3.74$ ppm (s, $3 \mathrm{H}) ;{ }^{13} \mathrm{C}-\mathrm{NMR}\left(\mathrm{CD}_{3} \mathrm{CN}\right): \delta=195.8,162.2,162.0,161.0,160.3,157.8,140.9,133.6,130.0$, 
129.1, 125.5, 123.4, 115.0, 114.8, 114.5, 108.9, 107.6, 106.0, 101.8, 94.1, 86.4, 70.9, 56.3, 56.2, 55.9, 55.7 ppm; HRMS (ESI): calcd for $\mathrm{C}_{33} \mathrm{H}_{30} \mathrm{O}_{7} \mathrm{Na}^{+}\left[\mathrm{M}+\mathrm{Na}^{+}\right] 561.1883$, found 561.1883.

\subsection{General procedure C (Preparation of benzofurans 19, Table 2)}

To a solution of benzyl ether $17(0.2 \mathrm{mmol})$ in THF $(2 \mathrm{~mL})$ at $0{ }^{\circ} \mathrm{C}$ was added LiTMP $(0.5 \mathrm{M}$ in THF, $2 \mathrm{~mL}, 1.0 \mathrm{mmol}$ ). The resulting mixture was stirred at $0{ }^{\circ} \mathrm{C}$ and the progress was monitored by TLC analysis. Upon completion of the reaction ( $\sim 2 \mathrm{~h}$ for most cases), the reaction mixture was quenched with $\mathrm{NH}_{4} \mathrm{Cl}$ ( $5 \mathrm{~mL}$, sat. aq.). The layers were separated and the aqueous layer was extracted with EtOAc $(3 \times 5 \mathrm{~mL})$. The combined organic layers were washed with brine $(10 \mathrm{~mL})$, dried $\left(\mathrm{Na}_{2} \mathrm{SO}_{4}\right)$ and concentrated in vacuo to afford crude tertiary alcohol 18, which was used directly without further purification. To a solution of the crude tertiary alcohol $\mathbf{1 8}$ (obtained as above) in $\mathrm{CH}_{2} \mathrm{Cl}_{2}(3 \mathrm{~mL})$ at $23{ }^{\circ} \mathrm{C}$ was added $p-\mathrm{TsOH} \cdot \mathrm{H}_{2} \mathrm{O}(38 \mathrm{mg}, 0.2 \mathrm{mmol})$. The resulting mixture was stirred for $1 \mathrm{~h}$ before it was quenched with $\mathrm{NaHCO}_{3}(3 \mathrm{~mL}$, sat. aq. $)$. The layers were separated and the aqueous layer was extracted with $\mathrm{CH}_{2} \mathrm{Cl}_{2}(3 \times 5 \mathrm{~mL})$. The combined organic layers were washed with brine $(5 \mathrm{~mL})$, dried $\left(\mathrm{Na}_{2} \mathrm{SO}_{4}\right)$ and concentrated in vacuo. Flash column chromatography (silica gel) afforded the desired benzofuran 19. Using this general procedure the following compounds were prepared:

6-Methoxy-2,3-diphenylbenzofuran (19a). From benzyl ether 17a. Flash column chromatography (silica gel, hexanes-EtOAc 4:1) afforded benzofuran 19a $(50 \mathrm{mg}, 83 \%)$ as a pale yellow oil. All physical properties of this compound were identical to those reported in literature [21].

(E)-3-(3,5-Dimethoxyphenyl)-6-methoxy-4-(4-methoxystyryl)-2-phenylbenzofuran (19b). From benzyl ether 17b. Flash column chromatography (silica gel, hexanes-EtOAc 4:1) afforded benzofuran 19b (70 mg, 71\%) as a yellow oil. 19b: $R_{\mathrm{f}}=0.42$ (silica gel, hexanes-EtOAc 2:1); IR (film) $v_{\max } 1601$, 1510, 1420, 1249, 1204, 1143, 1064, 1033, 808, $693 \mathrm{~cm}^{-1} ;{ }^{1} \mathrm{H}-\mathrm{NMR}\left(\mathrm{CD}_{3} \mathrm{CN}\right): \delta=7.58-7.57$ (m, 2 H), 7.32-7.25 (m, $3 \mathrm{H}), 7.15(\mathrm{~d}, J=2.4 \mathrm{~Hz}, 1 \mathrm{H}), 7.06(\mathrm{~d}, J=2.4 \mathrm{~Hz}, 1 \mathrm{H}), 7.01(\mathrm{~d}, J=16.2 \mathrm{~Hz}, 1 \mathrm{H})$, $6.99(\mathrm{~d}, J=8.4 \mathrm{~Hz}, 2 \mathrm{H}), 6.83(\mathrm{~d}, J=9.0 \mathrm{~Hz}, 2 \mathrm{H}), 6.79(\mathrm{~d}, J=16.2 \mathrm{~Hz}, 1 \mathrm{H}), 6.72(\mathrm{t}, J=2.4 \mathrm{~Hz}, 1 \mathrm{H})$, $6.65(\mathrm{~d}, J=2.4 \mathrm{~Hz}, 2 \mathrm{H}), 3.88(\mathrm{~s}, 3 \mathrm{H}), 3.77(\mathrm{~s}, 3 \mathrm{H}), 3.74 \mathrm{ppm}(\mathrm{s}, 6 \mathrm{H}) ;{ }^{13} \mathrm{C}-\mathrm{NMR}\left(\mathrm{CD}_{3} \mathrm{CN}\right): \delta=163.1$, $160.8,159.9,156.3,150.6,138.0,133.6,131.9,131.2,130.1,129.8,129.3,128.8,127.1,123.4,122.8$, 119.3, 115.3, 109.9, 108.0, 101.0, 96.1, 56.8, 56.6, 56.3 ppm; HRMS (ESI): calcd for $\mathrm{C}_{32} \mathrm{H}_{28} \mathrm{O}_{5} \mathrm{Na}^{+}[\mathrm{M}$ $\left.+\mathrm{Na}^{+}\right]$515.1829, found 515.1837.

(E)-2-(4-Bromophenyl)-3-(3,5-dimethoxyphenyl)-6-methoxy-4-(4-methoxystyryl)benzofuran (19c). From benzyl ether 17c. The desired benzofuran 19c was not obtained in this reaction.

(E)-3-(3,5-Dimethoxyphenyl)-6-methoxy-2-(4-methoxyphenyl)-4-(4-methoxystyryl)benzofuran

(19d). From benzyl ether 17d. Flash column chromatography (silica gel, hexanes:-EtOAc 2:1) afforded benzofuran 19d $(91 \mathrm{mg}, 87 \%)$ as a yellow solid. 19d: $R_{\mathrm{f}}=0.52$ (silica gel, hexanes-EtOAc 2:1); m.p. $=61-62{ }^{\circ} \mathrm{C}$ (hexanes-EtOAc); IR (film) $v_{\max } 2936,1603,1509,1250,1153,1143,1033,833,808$ $\mathrm{cm}^{-1}$; ${ }^{1} \mathrm{H}-\mathrm{NMR}\left(\mathrm{CD}_{3} \mathrm{CN}\right): \delta=7.43(\mathrm{~d}, J=8.4 \mathrm{~Hz}, 2 \mathrm{H}), 7.07(\mathrm{~d}, J=2.4 \mathrm{~Hz}, 1 \mathrm{H}), 6.95-6.89(\mathrm{~m}, 4 \mathrm{H})$, 
6.78-6.75 (m, $5 \mathrm{H}), 6.68$ (t, $J=2.4 \mathrm{~Hz}, 1 \mathrm{H}), 6.59$ (d, J = 2.4 Hz, $2 \mathrm{H}), 3.82$ (s, $3 \mathrm{H}), 3.73$ (s, $3 \mathrm{H})$, $3.70(\mathrm{~s}, 6 \mathrm{H}), 3.69 \mathrm{ppm}(\mathrm{s}, 3 \mathrm{H}) ;{ }^{13} \mathrm{C}-\mathrm{NMR}\left(\mathrm{CD}_{3} \mathrm{CN}\right): \delta=161.6,159.4,159.3,158.1,154.8,149.4$, 137.0, 131.8, 130.0, 128.4, 127.4, 127.3, 123.1, 122.4, 121.7, 116.2, 113.9, 113.8, 108.7, 106.4, 99.5, 94.7, 55.3, 55.2, 54.9, 54.8 ppm; HRMS (ESI): calcd for $\mathrm{C}_{33} \mathrm{H}_{30} \mathrm{O}_{6} \mathrm{Na}^{+}\left[\mathrm{M}+\mathrm{Na}^{+}\right]$545.1934, found 545.1951.

(E)-3-(3,5-Dimethoxyphenyl)-2-(furan-2-yl)-6-methoxy-4-(4-methoxystyryl)benzofuran (19e). From furanyl ether 17e. Flash column chromatography (silica gel, hexanes-EtOAc 2:1) afforded benzofuran 19e (36 mg, 38\%) as a yellow oil. 19e: $R_{\mathrm{f}}=0.40$ (silica gel, hexanes-EtOAc 2:1); IR (film) $v_{\max } 2936$, 1600, 1510, 1421, 1250, 1152, 1064, $819 \mathrm{~cm}^{-1} ;{ }^{1} \mathrm{H}-\mathrm{NMR}\left(\mathrm{CD}_{3} \mathrm{CN}\right): \delta=7.50(\mathrm{~d}, J=1.2 \mathrm{~Hz}, 1 \mathrm{H}), 7.16$ $(\mathrm{d}, J=2.4 \mathrm{~Hz}, 1 \mathrm{H}), 7.06(\mathrm{~d}, J=2.4 \mathrm{~Hz}, 1 \mathrm{H}), 7.02(\mathrm{~d}, J=15.6 \mathrm{~Hz}, 1 \mathrm{H}), 7.01(\mathrm{~d}, J=8.4 \mathrm{~Hz}, 2 \mathrm{H})$, 6.84-6.82 (m, $3 \mathrm{H}), 6.70$ (t, $J=2.4 \mathrm{~Hz}, 1 \mathrm{H}), 6.62(\mathrm{~d}, J=2.4 \mathrm{~Hz}, 2 \mathrm{H}), 6.46(\mathrm{q}, J=1.8 \mathrm{~Hz}, 1 \mathrm{H}), 6.38$ $(\mathrm{d}, J=3.0 \mathrm{~Hz}, 1 \mathrm{H}), 3.88(\mathrm{~s}, 3 \mathrm{H}), 3.77(\mathrm{~s}, 3 \mathrm{H}), 3.76 \mathrm{ppm}(\mathrm{s}, 6 \mathrm{H}) ;{ }^{13} \mathrm{C}-\mathrm{NMR}\left(\mathrm{CD}_{3} \mathrm{CN}\right): \delta=162.2$, $160.4,159.5,156.1,146.2,143.9,143.4,136.3,133.1,130.7,129.8,128.3,123.0,121.5,114.9,112.5$, 109.4, 107.7, 100.6, 95.8, 56.4, 56.1, 55.8 ppm; HRMS (ESI): calcd for $\mathrm{C}_{30} \mathrm{H}_{26} \mathrm{O}_{6} \mathrm{Na}^{+}\left[\mathrm{M}+\mathrm{Na}^{+}\right]$ 505.1621, found 505.1603.

(E)-6-Methoxy-2-(4-methoxyphenyl)-4-(4-methoxystyryl)-3-phenylbenzofuran (19f). According to General Procedure $\mathrm{C}$ using benzyl ether 17f. Flash column chromatography (silica gel, hexanes-EtOAc 4:1) afforded benzofuran 19f (74 mg, 80\%) as a yellow oil. 19f: $R_{\mathrm{f}}=0.60$ (silica gel, hexanes-EtOAc 2:1); IR (film) $v_{\max } 1605,1511,1251,1175,1144,1033,833,702 \mathrm{~cm}^{-1} ;{ }^{1} \mathrm{H}-\mathrm{NMR}$ ( $d_{6}$-acetone): $\delta=7.62-7.59(\mathrm{~m}, 3 \mathrm{H}), 7.52-7.50(\mathrm{~m}, 2 \mathrm{H}), 7.45(\mathrm{~d}, J=9.0 \mathrm{~Hz}, 2 \mathrm{H}), 7.18(\mathrm{~d}, J=2.4 \mathrm{~Hz}, 1 \mathrm{H}), 7.09$ $(\mathrm{d}, J=2.4 \mathrm{~Hz}, 1 \mathrm{H}), 7.03(\mathrm{~d}, J=16.2 \mathrm{~Hz}, 1 \mathrm{H}), 6.96(\mathrm{~d}, J=8.4 \mathrm{~Hz}, 2 \mathrm{H}), 6.86(\mathrm{~d}, J=9.0 \mathrm{~Hz}, 2 \mathrm{H})$, $6.80(\mathrm{~d}, J=8.4 \mathrm{~Hz}, 2 \mathrm{H}), 6.73(\mathrm{~d}, J=16.2 \mathrm{~Hz}, 1 \mathrm{H}), 3.91(\mathrm{~s}, 3 \mathrm{H}), 3.77 \mathrm{ppm}(\mathrm{s}, 6 \mathrm{H}) ;{ }^{13} \mathrm{C}-\mathrm{NMR}\left(d_{6^{-}}\right.$ acetone): $\delta=159.5,159.5,158.4,155.0,149.7,135.1,131.9,130.7,129.9,129.3,128.6,128.0,127.6$, 127.3, 123.2, 121.9, 121.8, 116.4, 113.8, 113.8, 106.5, 94.7, 55.2, 54.7, 54.6 ppm; HRMS (ESI): calcd for $\mathrm{C}_{31} \mathrm{H}_{26} \mathrm{O}_{4} \mathrm{Na}^{+}\left[\mathrm{M}+\mathrm{Na}^{+}\right]$485.1723, found 485.1713.

(E)-6-Methoxy-2-(4-methoxyphenyl)-4-(4-methoxystyryl)-3-(3,4,5-trimethoxyphenyl)benzofuran (19g). According to General Procedure C using benzyl ether 17g. Flash column chromatography (silica gel, hexanes:EtOAc 2:1) afforded benzofuran $19 \mathrm{~g}(94 \mathrm{mg}, 85 \%)$ as a yellow oil. 19g: $R_{\mathrm{f}}=0.34$ (silica gel, hexanes:EtOAc 2:1); IR (film) $v_{\max } 2936,1604,1511,1409,1250,1126,1033,838 \mathrm{~cm}^{-1}$; ${ }^{1} \mathrm{H}-\mathrm{NMR}$ $\left(\mathrm{CD}_{3} \mathrm{CN}\right): \delta=7.50(\mathrm{~d}, J=9.0 \mathrm{~Hz}, 2 \mathrm{H}), 7.14(\mathrm{~d}, J=1.8 \mathrm{~Hz}, 1 \mathrm{H}), 7.05-6.99(\mathrm{~m}, 4 \mathrm{H}), 6.86(\mathrm{~d}$, $J=9.0 \mathrm{~Hz}, 2 \mathrm{H}), 6.81(\mathrm{~d}, J=16.2 \mathrm{~Hz}, 1 \mathrm{H}), 6.78(\mathrm{~d}, J=9.0 \mathrm{~Hz}, 2 \mathrm{H}), 6.75(\mathrm{~s}, 2 \mathrm{H}), 3.88(\mathrm{~s}, 3 \mathrm{H}), 3.86$ $(\mathrm{s}, 3 \mathrm{H}), 3.76(\mathrm{~s}, 3 \mathrm{H}), 3.75(\mathrm{~s}, 3 \mathrm{H}), 3.68 \mathrm{ppm}(\mathrm{s}, 6 \mathrm{H}) ;{ }^{13} \mathrm{C}-\mathrm{NMR}\left(\mathrm{CD}_{3} \mathrm{CN}\right): \delta=159.5,159.4,158.2$, 154.8, 154.0, 149.7, 137.9, 132.0, 130.1, 130.0, 128.8, 127.4, 127.3, 123.2, 122.3, 121.7, 116.4, 114.0, 113.9, 107.9, 106.6, 94.8, 60.3, 55.9, 55.4, 54.9, 54.9 ppm; HRMS (ESI): calcd for $\mathrm{C}_{34} \mathrm{H}_{32} \mathrm{O}_{7} \mathrm{Na}^{+}[\mathrm{M}+$ $\left.\mathrm{Na}^{+}\right]$575.2040, found 575.2048.

(E)-3-(3,4-Dimethoxyphenyl)-6-methoxy-2-(4-methoxyphenyl)-4-(4-methoxystyryl)benzofuran (19h). From benzyl ether 17h. Flash column chromatography (silica gel, hexanes-EtOAc 2:1) afforded benzofuran 19h (85 mg, 81\%) as a yellow oil. 19h: $R_{\mathrm{f}}=0.35$ (silica gel, hexanes-EtOAc 2:1); IR (film) 
$v_{\max } 2923,1604,1509,1247,1138,1026,833,734 \mathrm{~cm}^{-1} ;{ }^{1} \mathrm{H}-\mathrm{NMR}\left(\mathrm{CD}_{3} \mathrm{CN}\right): \delta=7.49(\mathrm{~d}, J=9.0 \mathrm{~Hz}$, $2 \mathrm{H}), 7.12(\mathrm{~d}, J=1.8 \mathrm{~Hz}, 1 \mathrm{H}), 7.08(\mathrm{~d}, J=8.4 \mathrm{~Hz}, 1 \mathrm{H}), 7.03(\mathrm{~d}, J=2.4 \mathrm{~Hz}, 1 \mathrm{H}), 7.02(\mathrm{~d}, J=2.4 \mathrm{~Hz}$, $1 \mathrm{H}), 6.99-6.96(\mathrm{~m}, 4 \mathrm{H}), 6.85(\mathrm{~d}, J=9.0 \mathrm{~Hz}, 2 \mathrm{H}), 6.79$ (d, $J=9.0 \mathrm{~Hz}, 2 \mathrm{H}), 6.73$ (d, J = 16.2 Hz, $1 \mathrm{H}), 3.92(\mathrm{~s}, 3 \mathrm{H}), 3.88(\mathrm{~s}, 3 \mathrm{H}), 3.77(\mathrm{~s}, 3 \mathrm{H}), 3.76(\mathrm{~s}, 3 \mathrm{H}), 3.67 \mathrm{ppm}(\mathrm{s}, 3 \mathrm{H}) ;{ }^{13} \mathrm{C}-\mathrm{NMR}\left(\mathrm{CD}_{3} \mathrm{CN}\right)$ : $\delta=159.4,159.4,158.2,154.8,149.9,149.8,149.3,132.0,130.0,128.5,127.5,127.3,126.9,123.3$, $122.9,122.3,122.0,116.3,114.2,113.9,113.9,112.2$, 106.4, 94.8, 55.6, 55.5, 55.4, 54.9 ppm; HRMS (ESI): calcd for $\mathrm{C}_{33} \mathrm{H}_{30} \mathrm{O}_{6} \mathrm{Na}^{+}\left[\mathrm{M}+\mathrm{Na}^{+}\right]$545.1934, found 545.1946.

3-(3,5-Dimethoxyphenyl)-6-methoxy-2-(4-methoxyphenyl)-4-((4-methoxyphenyl)ethynyl)benzofuran (19i). From benzyl ether 17i. Flash column chromatography (silica gel, hexanes-EtOAc 4:1) afforded benzofuran 19i ( $88 \mathrm{mg}, 85 \%$ ) as a yellow oil. 19i: $R_{\mathrm{f}}=0.70$ (silica gel, hexanes-EtOAc 2:1); IR (film) $\nu_{\max } 2935,1604,1510,1485,1248,1152,1035,831 \mathrm{~cm}^{-1} ;{ }^{1} \mathrm{H}-\mathrm{NMR}\left(\mathrm{CD}_{3} \mathrm{CN}\right): \delta=7.47(\mathrm{~d}, J=9.0 \mathrm{~Hz}$, $2 \mathrm{H}), 7.16(\mathrm{~d}, J=2.4 \mathrm{~Hz}, 1 \mathrm{H}), 7.02(\mathrm{~d}, J=8.4 \mathrm{~Hz}, 2 \mathrm{H}), 6.99(\mathrm{~d}, J=2.4 \mathrm{~Hz}, 1 \mathrm{H}), 6.87$ (d, $J=9.0 \mathrm{~Hz}$, $2 \mathrm{H}), 6.85(\mathrm{~d}, J=8.4 \mathrm{~Hz}, 2 \mathrm{H}), 6.64(\mathrm{~d}, J=2.4 \mathrm{~Hz}, 2 \mathrm{H}), 6.56(\mathrm{t}, J=2.4 \mathrm{~Hz}, 1 \mathrm{H}), 3.87$ (s, $3 \mathrm{H}), 3.79$ $(\mathrm{s}, 3 \mathrm{H}), 3.77(\mathrm{~s}, 3 \mathrm{H}), 3.70 \mathrm{ppm}(\mathrm{s}, 6 \mathrm{H}) ;{ }^{13} \mathrm{C}-\mathrm{NMR}\left(\mathrm{CD}_{3} \mathrm{CN}\right): \delta=161.8,160.7,160.6,158.5,155.4$, 151.5, 135.8, 133.7, 128.6, 123.9, 123.7, 117.3, 116.4, 116.0, 115.5, 114.8, 114.7, 109.9, 100.4, 97.6, 94.6, 86.1, 56.5, 55.9, 55.8 ppm; HRMS (ESI): calcd for $\mathrm{C}_{33} \mathrm{H}_{28} \mathrm{O}_{6} \mathrm{Na}^{+}\left[\mathrm{M}+\mathrm{Na}^{+}\right] 543.1778$, found 543.1773 .

\subsection{General procedure D (Preparation of hexacyclic benzofurans 20, Table 3)}

To a solution of benzofuran $19(0.04 \mathrm{mmol})$ in $\mathrm{CH}_{2} \mathrm{Cl}_{2}(6 \mathrm{~mL})$ at $23{ }^{\circ} \mathrm{C}$ was added $p-\mathrm{TsOH} \cdot \mathrm{H}_{2} \mathrm{O}$ (22.8 mg, $0.12 \mathrm{mmol}$ ). The resulting mixture was heated to $40{ }^{\circ} \mathrm{C}$ and stirred for 8 hours before it was quenched with $\mathrm{NaHCO}_{3}(3 \mathrm{~mL}$, sat. aq.). The layers were separated and the aqueous layer was extracted with $\mathrm{CH}_{2} \mathrm{Cl}_{2}(3 \times 5 \mathrm{~mL})$. The combined organic layers were washed with brine $(5 \mathrm{~mL})$, dried $\left(\mathrm{Na}_{2} \mathrm{SO}_{4}\right)$ and concentrated in vacuo. Flash column chromatography (silica gel) afforded the desired hexacyclic benzofuran 20. The following compounds were prepared via this general procedure:

1,3,8-Trimethoxy-11-(4-methoxyphenyl)-5-phenyl-10,11-dihydrobenzo[6,7]cyclohepta[1,2,3-cd]benzofuran (20a). From benzofuran 19b. Flash column chromatography (silica gel, hexanes-EtOAc 4:1) afforded hexacyclic benzofuran 20a $\left(18.7 \mathrm{mg}, 95 \%\right.$ ) as a yellow oil. 20a: $R_{\mathrm{f}}=0.57$ (silica gel, hexanes-EtOAc 2:1); IR (film) $v_{\max }$ 2933, 1598, 1509, 1461, 1248,1144, 1065, 853, $696 \mathrm{~cm}^{-1}$; ${ }^{1} \mathrm{H}$ - NMR $\left(\mathrm{CD}_{3} \mathrm{CN}\right): \delta=7.66-7.64(\mathrm{~m}, 2 \mathrm{H}), 7.47-7.41(\mathrm{~m}, 3 \mathrm{H}), 7.01(\mathrm{~d}, J=8.4 \mathrm{~Hz}, 2 \mathrm{H}), 6.78(\mathrm{~d}$, $J=1.8 \mathrm{~Hz}, 1 \mathrm{H}), 6.71(\mathrm{~d}, J=1.8 \mathrm{~Hz}, 1 \mathrm{H}), 6.59(\mathrm{~d}, J=8.4 \mathrm{~Hz}, 2 \mathrm{H}), 6.58(\mathrm{~d}, J=2.4 \mathrm{~Hz}, 1 \mathrm{H}), 6.53(\mathrm{~d}$, $J=2.4 \mathrm{~Hz}, 1 \mathrm{H}), 5.44(\mathrm{~d}, J=5.4 \mathrm{~Hz}, 1 \mathrm{H}), 3.81(\mathrm{~s}, 3 \mathrm{H}), 3.78(\mathrm{~s}, 3 \mathrm{H}), 3.72(\mathrm{dd}, J=16.2,5.4 \mathrm{~Hz}, 1 \mathrm{H})$, $3.58(\mathrm{~s}, 3 \mathrm{H}), 3.44(\mathrm{~s}, 3 \mathrm{H}), 3.37 \mathrm{ppm}(\mathrm{d}, J=16.2 \mathrm{~Hz}, 1 \mathrm{H}) ;{ }^{13} \mathrm{C}-\mathrm{NMR}\left(\mathrm{CD}_{3} \mathrm{CN}\right): \delta=158.6,158.3$, 158.1, 157.2, 154.2, 150.6, 135.4, 134.1, 133.9, 131.7, 128.9, 128.7, 128.7, 128.3, 123.9, 119.8, 112.9, 112.6, 107.2, 97.8, 92.7, 55.8, 55.2, 54.5, 54.4, 36.7, 36.6 ppm; HRMS (ESI): calcd for $\mathrm{C}_{32} \mathrm{H}_{28} \mathrm{O}_{5} \mathrm{Na}^{+}$ $\left[\mathrm{M}+\mathrm{Na}^{+}\right]$515.1829, found 515.1835. 
hexacyclic benzofuran 20b (18.8 mg, 90\%) as a yellow oil. 20b: $R_{\mathrm{f}}=0.49$ (silica gel, hexanes-EtOAc 2:1); IR (film) $v_{\max } 2933,1599,1508,1460,1248,1143,1067,1032,834 \mathrm{~cm}^{-1}$; ${ }^{1} \mathrm{H}-\mathrm{NMR}\left(\mathrm{CD}_{3} \mathrm{CN}\right)$ : $\delta=7.57(\mathrm{~d}, J=9.0 \mathrm{~Hz}, 2 \mathrm{H}), 7.00-6.98(\mathrm{~m}, 4 \mathrm{H}), 6.75(\mathrm{~d}, J=1.8 \mathrm{~Hz}, 1 \mathrm{H}), 6.69(\mathrm{~d}, J=1.8 \mathrm{~Hz}, 1 \mathrm{H})$, $6.61(\mathrm{~d}, J=2.4 \mathrm{~Hz}, 1 \mathrm{H}), 6.58(\mathrm{~d}, J=9.0 \mathrm{~Hz}, 2 \mathrm{H}), 6.51(\mathrm{~d}, J=2.4 \mathrm{~Hz}, 1 \mathrm{H}), 5.43(\mathrm{~d}, J=6.0 \mathrm{~Hz}, 1 \mathrm{H})$, $3.83(\mathrm{~s}, 3 \mathrm{H}), 3.80$ (s, $3 \mathrm{H}), 3.76(\mathrm{~s}, 3 \mathrm{H}), 3.69$ (dd, $J=16.2,6.0 \mathrm{~Hz}, 1 \mathrm{H}), 3.57$ (s, $3 \mathrm{H}), 3.47$ (s, $3 \mathrm{H})$, $3.35 \mathrm{ppm}(\mathrm{d}, J=16.2 \mathrm{~Hz}, 1 \mathrm{H}) ;{ }^{13} \mathrm{C}-\mathrm{NMR}\left(\mathrm{CD}_{3} \mathrm{CN}\right): \delta=160.2,158.6,158.3,157.8,157.2,154.0$, $150.7,135.2,134.2,134.1,130.2$, 128.3, 123.9, 123.8, 119.9, 116.1, 114.1, 112.9, 112.3, 106.9, 97.6, 92.6, 55.8, 55.2, 55.1, 54.5, 54.4, 36.7, 36.6 ppm; HRMS (ESI): calcd for $\mathrm{C}_{33} \mathrm{H}_{30} \mathrm{O}_{6} \mathrm{Na}^{+}\left[\mathrm{M}+\mathrm{Na}^{+}\right]$ 545.1934 , found 545.1948 .

5-(Furan-2-yl)-1,3,8-trimethoxy-11-(4-methoxyphenyl)-10,11-dihydrobenzo[6,7]cyclohepta[1,2,3-cd]benzofuran (20c). From benzofuran 19e. The desired product 20c was not obtained in this reaction.

8-Methoxy-5,11-bis(4-methoxyphenyl)-10,11-dihydrobenzo[6,7]cyclohepta[1,2,3-cd]benzofuran (20d). From benzofuran 19f. The desired product 20d was not obtained in this reaction.

1,2,3,8-Tetramethoxy-5,11-bis(4-methoxyphenyl)-10,11-dihydrobenzo[6,7]cyclohepta[1,2,3-cd]benzofuran (20e). From benzofuran 19g. Flash column chromatography (silica gel, hexanes-EtOAc 4:1) afforded hexacyclic benzofuran $20 \mathrm{e}(20.3 \mathrm{mg}, 92 \%)$ as a yellow oil. $20 \mathrm{e}: R_{\mathrm{f}}=0.48$ (silica gel, hexanesEtOAc 2:1); IR (film) $v_{\max } 2933,1611,1508,1316,1248,1143,1037,835 \mathrm{~cm}^{-1} ;{ }^{1} \mathrm{H}-\mathrm{NMR}\left(\mathrm{CD}_{3} \mathrm{CN}\right): \delta$ $=7.60(\mathrm{~d}, J=8.4 \mathrm{~Hz}, 2 \mathrm{H}), 7.02-7.00(\mathrm{~m}, 4 \mathrm{H}), 6.89(\mathrm{~s}, 1 \mathrm{H}), 6.75(\mathrm{~s}, 1 \mathrm{H}), 6.69(\mathrm{~s}, 1 \mathrm{H}), 6.58(\mathrm{~d}$, $J=8.4 \mathrm{~Hz}, 2 \mathrm{H}), 5.31(\mathrm{~d}, J=5.4 \mathrm{~Hz}, 1 \mathrm{H}), 3.84(\mathrm{~s}, 3 \mathrm{H}), 3.83(\mathrm{~s}, 3 \mathrm{H}), 3.76(\mathrm{~s}, 3 \mathrm{H}), 3.74(\mathrm{dd}, J=16.2$, $5.4 \mathrm{~Hz}, 1 \mathrm{H}), 3.73(\mathrm{~s}, 3 \mathrm{H}), 3.40(\mathrm{~d}, J=16.2 \mathrm{~Hz}, 1 \mathrm{H}), 3.39 \mathrm{ppm}(\mathrm{s}, 3 \mathrm{H}) ;{ }^{13} \mathrm{C}-\mathrm{NMR}\left(\mathrm{CD}_{3} \mathrm{CN}\right)$ : $\delta=160.2$, 157.9, 157.2, 154.0, 152.2, 151.2, 150.1, 141.4, 134.9, 134.1, 130.1, 129.4, 128.3, 128.0, 123.9, 119.9, 115.8, 114.1, 112.9, 112.4, 110.2, 92.7, 61.2, 60.2, 55.2, 55.1, 54.8, 54.5, 38.0, 36.8 ppm; HRMS (ESI): calcd for $\mathrm{C}_{33} \mathrm{H}_{30} \mathrm{O}_{6} \mathrm{Na}^{+}\left[\mathrm{M}+\mathrm{Na}^{+}\right] 545.1934$, found 545.1948.

2,3,8-Trimethoxy-5,11-bis(4-methoxyphenyl)-10,11-dihydrobenzo[6,7]cyclohepta[1,2,3-cd]benzofuran (20f). From benzofuran 19h. Flash column chromatography (silica gel, hexanes-EtOAc 2:1) afforded hexacyclic benzofuran $20 f(18.8 \mathrm{mg}, 90 \%)$ as a yellow oil. 20f: $R_{\mathrm{f}}=0.35$ (silica gel, hexanes-EtOAc 2:1); IR (film) $v_{\max } 2934,1610,1511,1499,1251,1177,1143,1035,834,793 \mathrm{~cm}^{-1}$; ${ }^{1} \mathrm{H}-\mathrm{NMR}$ $\left(\mathrm{CD}_{3} \mathrm{CN}\right): \delta=8.07(\mathrm{~d}, J=9.0 \mathrm{~Hz}, 2 \mathrm{H}), 7.23(\mathrm{~s}, 1 \mathrm{H}), 7.08(\mathrm{~d}, J=9.0 \mathrm{~Hz}, 2 \mathrm{H}), 6.86(\mathrm{~d}, J=8.4 \mathrm{~Hz}$, $2 \mathrm{H}), 6.70(\mathrm{~d}, J=8.4 \mathrm{~Hz}, 2 \mathrm{H}), 6.64(\mathrm{~d}, J=2.4 \mathrm{~Hz}, 1 \mathrm{H}), 6.57(\mathrm{~s}, 1 \mathrm{H}), 6.21(\mathrm{~d}, J=2.4 \mathrm{~Hz}, 1 \mathrm{H}), 4.63$ $(\mathrm{dd}, J=6.6,2.4 \mathrm{~Hz}, 1 \mathrm{H}), 3.90(\mathrm{~s}, 3 \mathrm{H}), 3.74(\mathrm{~s}, 3 \mathrm{H}), 3.69(\mathrm{~s}, 3 \mathrm{H}), 3.66(\mathrm{~s}, 3 \mathrm{H}), 3.65$ (s, $3 \mathrm{H}), 3.62$ $(\mathrm{dd}, J=14.4,2.4 \mathrm{~Hz}, 1 \mathrm{H}), 3.15 \mathrm{ppm}(\mathrm{dd}, J=14.4,6.6 \mathrm{~Hz}, 1 \mathrm{H}) ;{ }^{13} \mathrm{C}-\mathrm{NMR}\left(\mathrm{CD}_{3} \mathrm{CN}\right): \delta=193.6$, 165.3, 165.0, 162.1 158.8, 153.5, 150.5, 148.4, 139.9, 139.6, 136.3, 132.9, 131.8, 130.4, 127.8, 122.6, 114.9, 114.6, 114.1, 113.4, 112.7, 107.6, 56.3, 56.3, 56.2, 56.1, 55.6, 49.7, 41.4 ppm; HRMS (ESI): calcd for $\mathrm{C}_{33} \mathrm{H}_{30} \mathrm{O}_{6} \mathrm{Na}^{+}\left[\mathrm{M}+\mathrm{Na}^{+}\right]$545.1934, found 545.1943.

1,3,8-Trimethoxy-5,11-bis(4-methoxyphenyl)benzo[6,7]cyclohepta[1,2,3-cd]benzofuran (20g). From benzofuran 19i. Flash column chromatography (silica gel, hexanes-EtOAc 4:1) afforded hexacyclic benzofuran $20 \mathrm{~g}(19.8 \mathrm{mg}, 95 \%)$ as a yellow oil. $20 \mathrm{~g}$ : $R_{\mathrm{f}}=0.70$ (silica gel, hexanes-EtOAc 2:1); IR 
(film) $v_{\max } 2919,1738,1606,1505,1462,1365,1247,1199,833 \mathrm{~cm}^{-1} ;{ }^{1} \mathrm{H}-\mathrm{NMR}\left(\mathrm{CD}_{3} \mathrm{CN}\right): \delta=7.82$ $(\mathrm{d}, J=9.0 \mathrm{~Hz}, 2 \mathrm{H}), 7.21(\mathrm{~d}, J=9.0 \mathrm{~Hz}, 2 \mathrm{H}), 7.08(\mathrm{~d}, J=9.0 \mathrm{~Hz}, 2 \mathrm{H}), 6.86(\mathrm{~d}, J=9.0 \mathrm{~Hz}, 2 \mathrm{H}), 6.78$ $(\mathrm{d}, J=1.8 \mathrm{~Hz}, 1 \mathrm{H}), 6.64(\mathrm{~d}, J=1.8 \mathrm{~Hz}, 1 \mathrm{H}), 6.62$ (d, $J=2.4 \mathrm{~Hz}, 1 \mathrm{H}), 6.59$ (s, $1 \mathrm{H}), 6.29$ (d, $J=2.4$ $\mathrm{Hz}, 1 \mathrm{H}), 3.86(\mathrm{~s}, 3 \mathrm{H}), 3.80(\mathrm{~s}, 3 \mathrm{H}), 3.78(\mathrm{~s}, 3 \mathrm{H}), 3.43(\mathrm{~s}, 3 \mathrm{H}), 3.17 \mathrm{ppm}(\mathrm{s}, 3 \mathrm{H}) ;{ }^{13} \mathrm{C}-\mathrm{NMR}$ $\left(\mathrm{CD}_{3} \mathrm{CN}\right): \delta=161.4,161.4,161.3,159.8,158.8,155.0,151.9,141.9,141.7,138.2,133.8,132.4$, 131.0, 127.4, 127.1, 125.0, 120.6, 116.4, 115.1, 114.1, 111.9, 106.2, 100.3, 94.6, 56.2, 56.0, 56.0, 55.7, 55.3 ppm; HRMS (ESI): calcd for $\mathrm{C}_{33} \mathrm{H}_{30} \mathrm{O}_{6} \mathrm{Na}^{+}\left[\mathrm{M}+\mathrm{Na}^{+}\right]$543.1784, found 543.1745.

\subsection{One-pot preparation of hexacyclic benzofuran $20 \mathbf{b}$}

To a solution of benzyl ketone $\mathbf{1 7 d}(100 \mathrm{mg}, 0.185 \mathrm{mmol})$ in $\mathrm{THF}(3 \mathrm{~mL})$ at $0{ }^{\circ} \mathrm{C}$ was added LiTMP (0.5 M in THF, $1.9 \mathrm{~mL}, 0.93 \mathrm{mmol})$. The resulting mixture was stirred at $0{ }^{\circ} \mathrm{C}$ for $30 \mathrm{~min}$ before it was quenched with $\mathrm{NH}_{4} \mathrm{Cl}(5 \mathrm{~mL}$, sat. aq.). The layers were separated and the aqueous layer was extracted with EtOAc $(3 \times 5 \mathrm{~mL})$. The combined organic layers were washed with brine $(5 \mathrm{~mL})$, dried $\left(\mathrm{Na}_{2} \mathrm{SO}_{4}\right)$ and concentrated in vacuo to afford crude alcohol, which was used directly without further purification. To a solution of the crude alcohol (obtained as above) in $\mathrm{CH}_{2} \mathrm{Cl}_{2}(3 \mathrm{~mL})$ at $23{ }^{\circ} \mathrm{C}$ was added $p$ - $\mathrm{TsOH} \cdot \mathrm{H}_{2} \mathrm{O}(105 \mathrm{mg}, 0.56 \mathrm{mmol})$. The resulting mixture was heated to $45{ }^{\circ} \mathrm{C}$ and stirred for $8 \mathrm{~h}$ before it was quenched with $\mathrm{NaHCO}_{3}(3 \mathrm{~mL}$, sat. aq.). The layers were separated and the aqueous layer was extracted with $\mathrm{CH}_{2} \mathrm{Cl}_{2}(3 \times 5 \mathrm{~mL})$. The combined organic layers were washed with brine $(5 \mathrm{~mL})$, dried $\left(\mathrm{Na}_{2} \mathrm{SO}_{4}\right)$ and concentrated in vacuo. Flash column chromatography (silica gel, hexanes-EtOAc 2:1) afforded the desired hexacyclic benzofuran $20 \mathbf{b}(77 \mathrm{mg}, 80 \%)$ as a yellow oil.

(E)-(3,5-Dimethoxyphenyl)(2-hydroxy-4-methoxy-6-(4-methoxystyryl)phenyl)methanone (16b'). To a

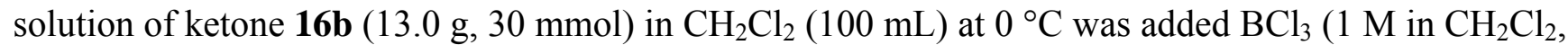
$45 \mathrm{~mL}, 45 \mathrm{mmol}$ ) dropwise. The resulting mixture was stirred for $1 \mathrm{~h}$ before it was quenched with $\mathrm{NaHCO}_{3}\left(50 \mathrm{~mL}\right.$, sat. aq.). The layers were separated and the aqueous layer was extracted with $\mathrm{CH}_{2} \mathrm{Cl}_{2}$ $(3 \times 50 \mathrm{~mL})$. The combined organic layers were washed with brine $(100 \mathrm{~mL})$, dried $\left(\mathrm{Na}_{2} \mathrm{SO}_{4}\right)$ and concentrated in vacuo. Flash column chromatography (silica gel, hexanes-EtOAc- $\mathrm{CH}_{2} \mathrm{Cl}_{2}$ 4:1:1) afforded phenol 16b' (12 g, 95\%) as a yellow solid. 16b': $R_{\mathrm{f}}=0.45$ (silica gel, hexanes-EtOAc 2:1); m.p. $=139-140{ }^{\circ} \mathrm{C}$ (hexanes-EtOAc); IR (film) $v_{\max } 2939,1600,1511,1457,1254,1204,1157,1064$, 840, $808 \mathrm{~cm}^{-1}$; ${ }^{1} \mathrm{H}-\mathrm{NMR}\left(\mathrm{CDCl}_{3}\right): \delta=11.5$ (br s, $\left.1 \mathrm{H}\right), 6.89(\mathrm{~d}, J=8.4 \mathrm{~Hz}, 2 \mathrm{H}), 6.74(\mathrm{~d}, J=8.4 \mathrm{~Hz}$, $2 \mathrm{H}), 6.72(\mathrm{~d}, J=2.4 \mathrm{~Hz}, 2 \mathrm{H}), 6.67(\mathrm{~d}, J=2.4 \mathrm{~Hz}, 1 \mathrm{H}), 6.65(\mathrm{~d}, J=16.2 \mathrm{~Hz}, 1 \mathrm{H}), 6.48(\mathrm{t}, J=2.4 \mathrm{~Hz}$, $1 \mathrm{H}), 6.47(\mathrm{~d}, J=2.4 \mathrm{~Hz}, 1 \mathrm{H}), 6.46(\mathrm{~d}, J=15.6 \mathrm{~Hz}, 1 \mathrm{H}), 3.88(\mathrm{~s}, 3 \mathrm{H}), 3.78(\mathrm{~s}, 3 \mathrm{H}), 3.68 \mathrm{ppm}(\mathrm{s}$, $6 \mathrm{H}) ;{ }^{13} \mathrm{C}-\mathrm{NMR}\left(\mathrm{CDCl}_{3}\right): \delta=200.1,164.7,164.6,160.6,159.4,142.7,142.7,130.0,129.5,127.7$, 126.9, 113.8, 113.3, 106.9, 106.3, 104.4, 99.9, 55.6, 55.2 ppm; HRMS (ESI): calcd for $\mathrm{C}_{25} \mathrm{H}_{24} \mathrm{O}_{6} \mathrm{Na}^{+}$ $\left[\mathrm{M}+\mathrm{Na}^{+}\right] 443.1465$, found 443.1454 .

3-(3,5-Dimethoxyphenyl)-6-methoxy-2-(4-methoxyphenyl)-4-(3-(4-methoxyphenyl)oxiran-2-yl)benzofuran (21). To a solution of benzofuran 19d $(4.20 \mathrm{~g}, 8.04 \mathrm{mmol})$ in DMSO $(50 \mathrm{~mL})$ and water $(10 \mathrm{~mL})$ at $0{ }^{\circ} \mathrm{C}$ was added NBS $(1.57 \mathrm{~g}, 8.84 \mathrm{mmol})$ in one portion. The resulting mixture was stirred for $0.5 \mathrm{~h}$ before it was quenching with $\mathrm{Na}_{2} \mathrm{~S}_{2} \mathrm{O}_{3}(50 \mathrm{~mL}$, sat. aq.). The layers were separated and the aqueous layer was extracted with $\mathrm{Et}_{2} \mathrm{O}(3 \times 100 \mathrm{~mL})$. The combined organic layers were washed with brine 
$(100 \mathrm{~mL})$, dried $\left(\mathrm{Na}_{2} \mathrm{SO}_{4}\right)$ and concentrated in vacuo to afford the crude bromohydrin, which was use directly without further purification. To a solution of crude bromohydrin (obtained as above) in $\mathrm{Et}_{2} \mathrm{O}$ $(100 \mathrm{~mL})$ at $23{ }^{\circ} \mathrm{C}$ were added $\mathrm{NaOH}(4 \mathrm{M}$, aq., $30 \mathrm{~mL})$ and $\mathrm{PhEt}_{3} \mathrm{NCl}$ (1.83 g, $\left.8.04 \mathrm{mmol}\right)$. The resulting mixture was stirred for $2 \mathrm{~h}$ before the layers were separated, and the aqueous layer was extracted with $\mathrm{Et}_{2} \mathrm{O}(3 \times 100 \mathrm{~mL})$. The combined organic layers were washed with brine $(100 \mathrm{~mL})$, dried $\left(\mathrm{Na}_{2} \mathrm{SO}_{4}\right)$ and concentrated in vacuo. Flash column chromatography (silica gel, hexanes-EtOAc 2:1) afforded epoxide 21 (3.25 g, 75\%, over the two steps) as a yellow solid. 21: $R_{\mathrm{f}}=0.55$ (silica gel, hexanes-EtOAc 2:1); m.p. $=147-148{ }^{\circ} \mathrm{C}$ (hexanes $/ \mathrm{CH}_{2} \mathrm{Cl}_{2}$ ); IR (film) $v_{\max } 2939,1738,1611,1587$, 1512, 1204, 1154, 1033, $832 \mathrm{~cm}^{-1} ;{ }^{1} \mathrm{H}-\mathrm{NMR}\left(\mathrm{CDCl}_{3}\right): \delta=7.47(\mathrm{~d}, J=9.0 \mathrm{~Hz}, 2 \mathrm{H}), 7.02(\mathrm{~d}, J=1.8$ $\mathrm{Hz}, 1 \mathrm{H}), 6.89$ (d, $J=8.4 \mathrm{~Hz}, 2 \mathrm{H}), 6.86(\mathrm{~d}, J=1.8 \mathrm{~Hz}, 1 \mathrm{H}), 6.80(\mathrm{~d}, J=8.4 \mathrm{~Hz}, 2 \mathrm{H}), 6.79$ (d, $J=9.0$ Hz, 2 H), 6.54 (br, 1 H), 6.31 (br, $1 \mathrm{H}), 6.18$ (t, J=1.8 Hz, $1 \mathrm{H}), 3.89$ (s, $3 \mathrm{H}), 3.83$ (s, $3 \mathrm{H}), 3.79$ (d, $J=2.4 \mathrm{~Hz}, 1 \mathrm{H}), 3.77$ (s, $3 \mathrm{H}), 3.74(\mathrm{br}, 3 \mathrm{H}), 3.53$ (d, $J=2.4 \mathrm{~Hz}, 1 \mathrm{H}), 3.30 \mathrm{ppm}(\mathrm{br}, 3 \mathrm{H}) ;{ }^{13} \mathrm{C}-\mathrm{NMR}$ $\left(\mathrm{CDCl}_{3}\right): \delta=161.0,159.7,159.2,158.2,154.2,149.7,136.0,131.5,128.5,127.2,126.9,123.2,122.5$, 115.4, 113.8, 113.6, 107.7 (br), 105.9, 99.7, 95.3, 63.1, 59.1, 55.8, 55.3, 55.2, 54.7 (br) ppm; HRMS (ESI): calcd for $\mathrm{C}_{33} \mathrm{H}_{30} \mathrm{O}_{7} \mathrm{Na}^{+}\left[\mathrm{M}+\mathrm{Na}^{+}\right]$561.1883, found 561.1898 .

Malibatol A (2): To a solution of epoxide 21 (100 $\mathrm{mg}, 0.19 \mathrm{mmol})$ in $\mathrm{CH}_{2} \mathrm{Cl}_{2}(30 \mathrm{~mL})$ at $-78{ }^{\circ} \mathrm{C}$ was added $\mathrm{BBr}_{3}\left(1.0 \mathrm{M}\right.$ in $\mathrm{CH}_{2} \mathrm{Cl}_{2}, 2.28 \mathrm{~mL}, 2.28 \mathrm{mmol}$ ). The resulting mixture was warmed to $23{ }^{\circ} \mathrm{C}$ and stirred for $2 \mathrm{~h}$ before it was quenched with $\mathrm{NaHCO}_{3}(10 \mathrm{~mL}$, sat. aq.). The layers were separated and the aqueous layer was extracted with EtOAc $(3 \times 20 \mathrm{~mL})$. The combined organic layers were washed with brine $(20 \mathrm{~mL})$, dried $\left(\mathrm{Na}_{2} \mathrm{SO}_{4}\right)$ and concentrated in vacuo. Flash column chromatography (silica gel, $\mathrm{CH}_{2} \mathrm{Cl}_{2}-\mathrm{MeOH}$ 9:1) afforded malibatol $(2,17.8 \mathrm{mg}, 20 \%)$ as a tan oil. Compound 2: $R_{\mathrm{f}}=0.23$ (silica gel, $\mathrm{CH}_{2} \mathrm{Cl}_{2}-\mathrm{MeOH}$ 9:1); IR (film) $v_{\max }$ 3323, 2918, 1612, 1510, 1433, 1366, 1231, 1139, $833 \mathrm{~cm}^{-1} ;{ }^{1} \mathrm{H}-\mathrm{NMR}\left(\mathrm{CD}_{3} \mathrm{OD}\right): \delta=7.45(\mathrm{~d}, J=8.6 \mathrm{~Hz}, 2 \mathrm{H}), 7.02(\mathrm{~d}, J=8.6 \mathrm{~Hz}, 2 \mathrm{H}), 7.01(\mathrm{~d}$, $J=2.4 \mathrm{~Hz}, 1 \mathrm{H}), 6.80(\mathrm{~d}, J=8.6 \mathrm{~Hz}, 2 \mathrm{H}), 6.57(\mathrm{dd}, J=2.4,1.2 \mathrm{~Hz}, 1 \mathrm{H}), 6.51(\mathrm{~d}, J=2.4 \mathrm{~Hz}, 1 \mathrm{H})$, $6.33(\mathrm{~d}, J=9.0 \mathrm{~Hz}, 2 \mathrm{H}), 6.30(\mathrm{~d}, J=2.4 \mathrm{~Hz}, 1 \mathrm{H}), 5.46$ (brs, $1 \mathrm{H}), 5.28 \mathrm{ppm}(\mathrm{m}, 1 \mathrm{H}) ;{ }^{13} \mathrm{C}-\mathrm{NMR}$ $\left(\mathrm{CD}_{3} \mathrm{OD}\right): \delta=159.1,157.4,156.7,156.2,155.3,155.1,151.2,139.6,135.8,133.4,130.9,130.6$, 124.6, 121.2, 119.0, 117.3, 116.4, 114.7, 109.9, 109.7, 102.1, 95.9, 74.8, 48.9 ppm; HRMS (ESI): calcd for $\mathrm{C}_{28} \mathrm{H}_{20} \mathrm{O}_{7} \mathrm{Na}^{+}\left[\mathrm{M}+\mathrm{Na}^{+}\right]$491.1101, found 491.1092.

Shoreaphenol (3): To a solution of malibatol A (2) $(5 \mathrm{mg}, 10.7 \mu \mathrm{mol})$ in THF $(1 \mathrm{~mL})$ at $23{ }^{\circ} \mathrm{C}$ was added PDC ( $4.8 \mathrm{mg}, 12.8 \mu \mathrm{mol})$. The resulting mixture was stirred for $1 \mathrm{~h}$ before it was quenched with $\mathrm{Na}_{2} \mathrm{~S}_{2} \mathrm{O}_{3}(1 \mathrm{~mL}$, sat. aq.). The layers were separated and the aqueous layer was extracted with EtOAc $(3 \times 2 \mathrm{~mL})$. The combined organic layers were washed with brine $(3 \mathrm{~mL})$, dried $\left(\mathrm{Na}_{2} \mathrm{SO}_{4}\right)$ and concentrated in vacuo. Flash column chromatography (silica gel, $\mathrm{CH}_{2} \mathrm{Cl}_{2}-\mathrm{MeOH}$ 5:1) afforded shoreaphenol (3, $2.3 \mathrm{mg}, 46 \%$ ) as a yellow oil. Compound 3: $R_{\mathrm{f}}=0.26$ (silica gel, $\mathrm{CH}_{2} \mathrm{Cl}_{2}-\mathrm{MeOH} 9: 1$ ); IR (film) $v_{\max } 3339,1738,1612,1366,1216,829 \mathrm{~cm}^{-1} ;{ }^{1} \mathrm{H}-\mathrm{NMR}$ ( $d_{6}$-acetone): $\delta=7.70$ (d, $J=8.4 \mathrm{~Hz}, 2 \mathrm{H}), 7.33(\mathrm{~d}, J=2.4 \mathrm{~Hz}, 1 \mathrm{H}), 7.04(\mathrm{~d}, J=1.8 \mathrm{~Hz}, 1 \mathrm{H}), 6.98(\mathrm{~d}, J=8.4 \mathrm{~Hz}, 2 \mathrm{H}), 6.85$ (d, $J=7.8 \mathrm{~Hz}, 2 \mathrm{H}), 6.70(\mathrm{~d}, J=2.4 \mathrm{~Hz}, 1 \mathrm{H}), 6.57(\mathrm{~d}, J=2.4 \mathrm{~Hz}, 1 \mathrm{H}), 6.55(\mathrm{~d}, J=8.4 \mathrm{~Hz}, 2 \mathrm{H}), 6.12$ (brs, $1 \mathrm{H}), 5.28 \mathrm{ppm}(\mathrm{m}, 1 \mathrm{H}) ;{ }^{13} \mathrm{C}$-NMR $\left(d_{6}\right.$-acetone): $\delta=196.3,159.5,158.3,157.7,156.4,156.1$, 154.9, 153.3, 135.2, 131.1, 131.0, 130.6, 128.5, 123.1, 122.4, 116.6, 116.4, 115.6, 114.0, 112.0, 109.0, 103.0, 102.4, 56.1 ppm; HRMS (ESI): calcd for $\mathrm{C}_{28} \mathrm{H}_{18} \mathrm{O}_{7} \mathrm{Na}^{+}\left[\mathrm{M}+\mathrm{Na}^{+}\right]$489.0950, found 489.0955. 


\section{Conclusions}

In conclusion, a modular and efficient entry to the dimeric resveratrol derived polyphenolic benzofurans has been developed, and applied to the total synthesis of malibatol A (2) and shoreaphenol (3). In view of the largely untapped potential of the polyphenolic secondary metabolites, the synthetic methodology described herein should find wide application in the chemical and biological investigations of this fascinating class of compounds.

\section{Acknowledgements}

We thank Doris Tan (ICES) for high resolution mass spectrometric (HRMS) assistance and Rong-Ji Sum (CSL-ICES) for his assistance in the biological studies. Financial support for this work was provided by A*STAR, Singapore.

\section{References and Notes}

1. Yang, C.S.; Lambert, J.D.; Sang, S. Antioxidative and anti-carcinogenic activities of tea polyphenols. Arch. Toxicol. 2009, 83, 11-21.

2. Bonfili, L.; Cecarini, V.; Amici, M.; Cuccioloni, M.; Angeletti, M.; Keller, J.N.; Eleuteri, A.M. Natural polyphenols as proteasome modulators and their role as anti-cancer compounds. FEBS J. 2008, 275, 5512-5526.

3. Lafay, S.; Gil-Izquierdo, A. Bioavailability of phenolic acids. Phytochem. Rev. 2008, 7, 301-311.

4. Habauzit, V.; Horcajada, M.-N. Phenolic phytochemicals and bone. Phytochem. Rev. 2008, 7, 313-344.

5. Halliwell, B. Are polyphenols antioxidants or pro-oxidants? What do we learn from cell culture and in vivo studies? Arch. Biochem. Biophys. 2008, 476, 107-112.

6. Korkina, L.G.; Pastore, S.; De Luca, C.; Kostyuk, V.A. Metabolism of plant polyphenols in the skin: Beneficial versus deleterious effects. Curr. Drug Metab. 2008, 9, 710-729.

7. Snyder, S.A.; Breazzano, S.P.; Ross, A.G.; Lin, Y.; Zografos, A.L. Total synthesis of diverse carbogenic complexity within the resveratrol class from a common building block. J. Am. Chem. Soc. 2009, 131, 1753-1765.

8. Snyder, S.A.; Zografos, L.; Lin, Y. Total synthesis of resveratrol-based natural products: A chemoselective solution. Angew. Chem. Int. Ed. 2007, 46, 8186-8191.

9. Nicolaou, K.C.; Wu, R.T.; Kang, Q.; Chen, D.Y.K. Total synthesis of hopeahainol A and hopeanol. Angew. Chem. Int. Ed. 2009, 48, 3340-3343.

10. Nicolaou K.C.; Kang Q.; Wu. R.T.; Lim C.S.; Chen D.Y.K. Total synthesis and biological evaluation of the resveratrol-derived polyphenol natural products hopeanol and hopeahainol A. $J$. Am. Chem. Soc. 2010, 132, 7540-7548.

11. Dai, J.-R.; Hallock, Y.F.; Cardellina, J.H., II; Boyd, M.R. HIV-Inhibitory and cytotoxic oligostilbenes from the leaves of Hopea malibato. J. Nat. Prod. 1998, 61, 351-353. 
12. Saraswathy, A.; Purushothaman, K.K.; Patra, A.; Dey, A.K.; Kundu, A.B. Shoreaphenol, a polyphenol from Shorea Robusta. Phytochemisry 1992, 31, 2561-2562.

13. Tanaka, T.; Ito, T.; Ido, Y.; Nakaya, K.; Iinuma, M.; Chelladurai, V. Hopeafuran and a C-glucosyl resveratrol isolated from stem wood of hopea utilis. Chem. Pharm. Bull. 2001, 49, 785-787.

14. Boutagy, J.; Thomas, R. Olefin synthesis with organic phosphonate carbanions. Chem. Rev. 1974, 74, 87-99.

15. Jones, G.; Stanforth, S.P. The vilsmeier reaction of non-aromatic compounds. Org. React. 1997, 49, 1-330.

16. Hughes, D.L. The mitsunobu reaction. Org. React. 1992, 42, 335-656.

17. Kraus, G.A.; Gupta, V. A new synthetic strategy for the synthesis of bioactive stilbene dimmers. A direct synthesis of amurensin. Tetrahedron Lett. 2009, 50, 7180.

18. Kraus, G.A.; Kim, I. Synthetic approach to malibatol A. Org. Lett. 2003, 5, 1191-1192.

19. Kim, I.; Choi, J. A versatile approach to oligostilbenoid natural products - synthesis of permethylated analogues of viniferifuran, malibatol A, and shoreaphenol. Org. Biomol. Chem. 2009, 7, 2788-2795.

20. Hajipour, A.R.; Zarei, A.; Khazdooz, L.; Ruoho, A.E. Simple and efficient procedure for the Friedel-Crafts acylation of aromatic compounds with carboxylic acids in the presence of $\mathrm{P}_{2} \mathrm{O}_{5} / \mathrm{Al}_{2} \mathrm{O}_{3}$ under heterogeneous conditions. Synth. Commun. 2009, 39, 2702-2722.

21. Brady, W.T.; Giang, Y.S.F.; Marchand, A.P.; Wu, A.H. Intramolecular [2+2] cycloadditions of ketenes to carbonyl groups. A novel synthesis of substituted benzofurans. J. Org. Chem. 1987, 52, $3457-3461$.

Sample Availability: Contact the authors.

(C) 2010 by the authors; licensee MDPI, Basel, Switzerland. This article is an open access article distributed under the terms and conditions of the Creative Commons Attribution license (http://creativecommons.org/licenses/by/3.0/). 